\title{
Synthesis of Planar Chiral Phosphapalladacycles by Highly Enantioselective Transcyclopalladation
}

\author{
Francesc X. Roca, Majid Motevalli and Christopher J. Richards \\ Department of Chemistry, Queen Mary, University of London, Mile End Road, London, E1 4NS, \\ $U K$

\section{Supplementary Material}

Index

Synthesis of 2-(dicyclohexylphosphino)phenylferrocene 5 S2

Synthesis of 2-(diphenylphosphino)phenylferrocene $6 . \quad$ S2

Synthesis of ${ }_{\mathrm{p}} S$-di- $\mu$-chlorobis[2-(2-dicyclohexylphosphino)-

phenylferroene- $C^{1}, P$ ) dipalladium(II) $7 \mathbf{b} . \quad \mathrm{S} 3$

Synthesis of ${ }_{\mathrm{p}} R$-di- $\mu$-chlorobis[2-(2-dicyclohexylphosphino)phenylferroene- $C^{1}, P$ ) dipalladium(II) $7 \mathbf{b}$. $\quad \mathrm{S} 4$

Determination of the enantiomeric excess of $\mathbf{7 b} . \quad \mathrm{S} 4$

Synthesis of ${ }_{\mathrm{p}} S$-di- $\mu$-chlorobis[2-(diphenylphosphino)-

$\begin{array}{ll}\left.\text { phenylferrocene- } C^{1}, P\right] \text { dipalladium } \mathbf{8 b} & \mathrm{S} 7\end{array}$

Synthesis of ${ }_{\mathrm{p}} R$-di- $\mu$-chlorobis[2-(diphenylphosphino)$\begin{array}{ll}\left.\text { phenylferrocene- } C^{1}, P\right] \text { dipalladium } \mathbf{8 b} & \mathrm{S} 7\end{array}$

$\begin{array}{ll}\text { Determination of the enantiomeric excess of } \mathbf{8 b} & \mathrm{S} 7\end{array}$

$\begin{array}{lr}\text { Isolation of } \mathbf{9 a} / \mathbf{b} \text {. } & \mathrm{S} 10\end{array}$

$\begin{array}{ll}\text { Crystallographic data for } \mathbf{7 b} . & \mathrm{S} 13\end{array}$

$\begin{array}{ll}\text { References } & \text { S41 }\end{array}$ 
General considerations. Petroleum ether refers to that fraction boiling in the range $40-60{ }^{\circ} \mathrm{C}$. THF was distilled from sodium benzophenone ketyl. Toluene was not specifically dried. Silica gel 40-63 $\mu \mathrm{m}$ (Merck) was used for chromatography. All reactions other than the transcyclopalladations were performed under nitrogen.

Synthesis of 2-(dicyclohexylphosphino)phenylferrocene 5. A solution of BuLi in hexanes $(0.63 \mathrm{~mL}, 1.46 \mathrm{mmol})$ was added slowly to 2-bromophenylferrocene ${ }^{1}(0.471 \mathrm{~g}, 1.38 \mathrm{mmol})$ in dry THF $(6 \mathrm{~mL})$ at $-78{ }^{\circ} \mathrm{C}$. The stirred solution was maintained at $-78{ }^{\circ} \mathrm{C}$ for $1 \mathrm{~h}$. Chlorodicyclohexylphosphine $(0.44 \mathrm{~mL}, 2.0 \mathrm{mmol})$ was added to the reaction mixture which was then allowed to warm to room temperature and stirred for a further 2 hours. The mixture was quenched with water and extracted with $\mathrm{CH}_{2} \mathrm{Cl}_{2}(2 \times 10 \mathrm{~mL})$. The combined organic layers were washed with brine, dried $\left(\mathrm{MgSO}_{4}\right)$ and the solvent removed in vacuo. The crude product was purified by column chromatography (25\% ethyl acetate/40-60 petroleum ether) to give 2(dicyclohexylphosphino)phenylferrocene as a bright orange oil which crystallised on standing $(0.447 \mathrm{~g}, 71 \%)$.

5: Mp 127-129 ${ }^{\circ} \mathrm{C}$. Anal. Found: C, 73.56; H 7.76. Calcd for $\mathrm{C}_{28} \mathrm{H}_{35} \mathrm{FeP}: \mathrm{C}, 73.36 ; \mathrm{H}, 7.70 .{ }^{1} \mathrm{H}$ NMR $\left(\delta, 270 \mathrm{MHz}, \mathrm{CDCl}_{3}\right)$ 0.86-1.25 (12H, m, PCy $), 1.40-1.75\left(10 \mathrm{H}, \mathrm{m}, \mathrm{PC} y_{2}\right), 4.06(5 \mathrm{H}, \mathrm{s}$, $\left.\mathrm{C}_{5} H_{5}\right), 4.20(2 \mathrm{H}, \mathrm{brs}, \mathrm{Fc}-\beta), 4.48(2 \mathrm{H}, \mathrm{brs}, \mathrm{Fc}-\alpha), 7.12(1 \mathrm{H}, \mathrm{t}, J$ 7, Ar), 7.24 (1H, t, $J$ 7, Ar), 7.33 $(1 \mathrm{H}, \mathrm{d}, J 7, \mathrm{Ar}), 7.85(1 \mathrm{H}, \mathrm{dd}, J 7,3, \mathrm{Ar}) .{ }^{13} \mathrm{C}\left\{{ }^{1} \mathrm{H}\right\} \mathrm{NMR}\left(\delta, 68 \mathrm{MHz}, \mathrm{CDCl}_{3}\right) 26.49$ (s, Cy), 27.24 (d, J 3, Cy), 27.39 (d, J 6, Cy), 29.41 (d, J 9, Cy), 30.41 (d, J 17, Cy), 34.75 (d, J 14, Cy), 67.47 (s, Fc- $\beta$ ), 69.44 (s, $C_{5} \mathrm{H}_{5}$ ), 72.3 (d, $J$ 10, Fc- $\alpha$ ), 89.10 (d, J 7, Fc-ipso), 125.36 (Ar), 127.78 (Ar), 131.88 (d, J 5, Ar), 132.34 (s, Ar), 134.87 (d, J 23, Ar), 146.02 (d, J 25, Ar-ipso). ${ }^{31} \mathrm{P}\left\{{ }^{1} \mathrm{H}\right\}$ NMR $\left(\delta, 109 \mathrm{MHz}, \mathrm{CDCl}_{3}\right)-11.81\left(-P \mathrm{Py}_{2}\right)$. MS (m/z, ES $) 459.4\left(\mathrm{MH}^{+}, 64 \%\right), 263.2(100)$.

Synthesis of 2-(diphenylphosphino)phenylferrocene 6. A solution of BuLi in hexanes (1.36 $\mathrm{mL}, 3.38 \mathrm{mmol})$ was added slowly to a solution of 2-bromophenylferrocene ${ }^{1}(1.00 \mathrm{~g}, 2.93$ mmol) in dry THF $(30 \mathrm{~mL})$ at $-78{ }^{\circ} \mathrm{C}$. The stirred solution was maintained at $-78{ }^{\circ} \mathrm{C}$ for $1.5 \mathrm{~h}$. Chlorodiphenylphosphine $(0.76 \mathrm{~mL}, 4.2 \mathrm{mmol})$ was added to the reaction mixture which was then allowed to warm to room temperature and stirred for a further 2 hours. The mixture was quenched with water and extracted with $\mathrm{CH}_{2} \mathrm{Cl}_{2}(2 \times 10 \mathrm{~mL})$. The combined organic layers were washed with brine, dried $\left(\mathrm{MgSO}_{4}\right)$ and the solvent removed in vacuo. The crude product was 
purified by column chromatography (10\% ethyl acetate/40-60 petroleum ether) to give 2(diphenylphosphino)phenylferrocene as deep red crystals (1.00 g, $76 \%$ ).

6: Mp 79-80 ${ }^{\circ} \mathrm{C}$. Anal. Found: C, 75.31; H 5.31. Calcd for $\mathrm{C}_{28} \mathrm{H}_{23} \mathrm{FeP}: \mathrm{C}, 75.35 ; \mathrm{H}, 5.19 .{ }^{1} \mathrm{H}$ NMR $\left(\delta, 270 \mathrm{MHz}, \mathrm{CDCl}_{3}\right) 4.07\left(5 \mathrm{H}, \mathrm{s}, \mathrm{C}_{5} H_{5}\right), 4.18$ (2H, brs, Fc- $\left.\beta\right), 4.42$ (2H, brs, Fc- $\left.\alpha\right), 6.83$ $(1 \mathrm{H}, \mathrm{ddd}, J 7,4,1, \mathrm{Ar}), 7.11(1 \mathrm{H}, \mathrm{td}, J 7,1, \mathrm{Ar}) 7.17-7.37(11 \mathrm{H}, \mathrm{m}), 7.94(1 \mathrm{H}, \mathrm{ddd}, J 7,4,1, \mathrm{Ar})$. ${ }^{13} \mathrm{C}\left\{{ }^{1} \mathrm{H}\right\}$ NMR $\left(\delta, 68 \mathrm{MHz}, \mathrm{CDCl}_{3}\right) 68.19(\mathrm{Fc}-\beta), 69.65\left(C_{5} \mathrm{H}_{5}\right), 71.32(\mathrm{~d}, J$ 8, Fc- $\alpha), 87.94(\mathrm{~d}, J$ 8, Fc-ipso), 126.41 (Ar), 128.42 (Ar), 128.50 (Ar), 128.59 (Ar), 131.46 (Ar), 131.54 (Ar), 134.05 (d, $J$ 20, Ar), 134.07 (Ar), 135.72 (d, $J$ 15, Ar), 138.09 (d, $J$ 12, Ar), 144.58 (d, $J ~ 26, A r)$. ${ }^{31} \mathrm{P}\left\{{ }^{1} \mathrm{H}\right\}$ NMR $\left(\delta, 109 \mathrm{MHz}, \mathrm{CDCl}_{3}\right)-11.63\left(-\mathrm{PPh}_{2}\right)$. MS $(\mathrm{m} / \mathrm{z}, \mathrm{CI}) 447.2\left(\mathrm{MH}^{+}, 50 \%\right), 263.1$ (70\%), $187(100)$.

\section{Synthesis of $\quad{ }_{p} S$-di- $\mu$-chlorobis[2-(2-dicyclohexylphosphino)phenylferroene-}

$C^{1}, P$ )dipalladium(II) 7 b. A round-bottomed flask containing a stirrer bar was charged with 2(dicyclohexylphosphino)phenylferrocene $5(0.460 \mathrm{~g}, 1.00 \mathrm{mmol})$ and di- $\mu$-acetatobis $\left[\left(\eta^{5}-(S, \mathrm{p} R)\right.\right.$ 2-(2'-(4'-methylethyl)oxazolinyl)cyclopentadienyl)( $\eta^{4}$-tetraphenylcyclobutadiene)cobalt- $\left.C^{1}, N^{3}\right]$ dipalladium $3 \mathrm{a}^{2}(0.794 \mathrm{~g}, 0.53 \mathrm{mmol})$. Addition of toluene $(20 \mathrm{~mL})$ gave a deep red homogeneous coloured solution which was heated to $70{ }^{\circ} \mathrm{C}$ and maintained at this temperature for 5 hours. On completion the reaction mixture was allowed to cool to room temperature and shaken for $10 \mathrm{~min}$. with a saturated solution of sodium chloride $(20 \mathrm{~mL})$. After separation, the aqueous phase was further extracted with $\mathrm{CH}_{2} \mathrm{Cl}_{2}(2 \times 10 \mathrm{~mL})$ and the combined organics were dried $\left(\mathrm{MgSO}_{4}\right)$, filtered, and the solvent removed in vacuo. Column chromatography $\left(\mathrm{SiO}_{2}, 1 \%\right.$ $\left.\mathrm{NEt}_{3}, \mathrm{CH}_{2} \mathrm{Cl}_{2}\right)$ gave first 1 as a bright yellow crystalline solid $(0.508 \mathrm{~g}, 81 \%)$ followed by the product phosphapalladacycle $\mathbf{7 b}$ as a bright orange-red foam $(0.598 \mathrm{~g}, 99 \%)$.

${ }_{\mathrm{p}} \boldsymbol{S}$-7b: Mp 208-215 ${ }^{\circ} \mathrm{C}$ (decomp.). Anal. Found: C, 55.90; H, 5.84\%. Calcd for $\mathrm{C}_{56} \mathrm{H}_{68} \mathrm{Cl}_{2} \mathrm{Fe}_{2} \mathrm{P}_{2} \mathrm{Pd}_{2}$ : C, 56.12; H, 5.72. $[\alpha]_{\mathrm{D}}{ }^{25}$-551 (c 0.1, $\left.\mathrm{CH}_{2} \mathrm{Cl}_{2}\right) .{ }^{1} \mathrm{H}$ NMR $(\delta, 270 \mathrm{MHz}$, $\mathrm{CDCl}_{3}$ ) Ratio of isomers 4:3: 0.8-2.35 (21H, m, Cy), 2.87 (1H, brs, Cy), 4.19 (brs, $\mathrm{C}_{5} H_{5}-$ major), 4.26 (brs, $\mathrm{C}_{5} H_{5}$ - minor), 4.41 (1H, brs, Fc), 4.63 (1H, brs, Fc), 4.70 (brs, Fc - major), 4.85 (brs, Fc - minor), $7.16(1 \mathrm{H}, \mathrm{t}, J 7, \mathrm{Ar}), 7.36(1 \mathrm{H}, \mathrm{t}, J 7, \mathrm{Ar}) 7.44(1 \mathrm{H}, \mathrm{t}, J 7, \mathrm{Ar}), 7.65(1 \mathrm{H}$, $\mathrm{dd}, J 7,3, \mathrm{Ar}) .{ }^{13} \mathrm{C}\left\{{ }^{1} \mathrm{H}\right\} \mathrm{NMR}\left(\delta, 68 \mathrm{MHz}, \mathrm{CDCl}_{3}\right) 25.9-39.0\left(\mathrm{~m}, \mathrm{Cy}_{2}\right), 67.4(\mathrm{Fc}), 69.7(\mathrm{Fc})$, $70.6(\mathrm{Fc}), 71.1\left(C_{5} \mathrm{H}_{5}\right), 73.0(\mathrm{~s}, \mathrm{Fc}), 75.5(\mathrm{Fc}), 80.23$ (d, $J$ 22, Fc), 83.5 (d, J 8, Fc), 119.7 (Ar), 120.4 (Ar), 124.2 (Ar), 124.3 (Ar), 126.4 (Ar), 127.7 (Ar), 127.9 (Ar), 128.1 (Ar), 129.3 (Ar), 
129.7 (Ar), 130.4 (Ar), 135.63 (s, Ar), 135.8 (Ar), 149.7 (Ar). ${ }^{31} \mathrm{P}\left\{{ }^{1} \mathrm{H}\right\}$ NMR $(\delta, 109 \mathrm{MHz}$, $\left.\mathrm{CDCl}_{3}\right) 38.85$ - minor, 39.75 - major $\left(\mathrm{PCy}_{2}\right)$. $\mathrm{MS}\left(\mathrm{m} / \mathrm{z}\right.$, ES) $564\left(1 / 2 \mathrm{M}^{+}-\mathrm{Cl} 10 \%\right), 457(100)$.

Synthesis of $\quad{ }_{\mathrm{p}} R$-di- $\mu$-chlorobis[2-(2-dicyclohexylphosphino)phenylferroene$\left.\boldsymbol{C}^{\mathbf{1}}, \boldsymbol{P}\right)$ dipalladium(II) $\mathbf{7 b}$. The same procedure described for the synthesis of ${ }_{\mathrm{p}} S-\mathbf{7 b}$ was employed using 2-(dicyclohexylphosphino)phenylferrocene 5 (0.460 g, $1.00 \mathrm{mmol})$, di- $\mu$ acetatobis $\left[\left(\eta^{5}-(S, p S)-2-\left(2^{\prime}-\left(4^{\prime}-\right.\right.\right.\right.$ tert-butyl $)$ oxazolinyl $)$ cyclopentadienyl $)\left(\eta^{4}-\right.$

tetraphenylcyclobutadiene)cobalt- $\left.C^{1}, N^{3^{\prime}}\right]$ dipalladium $4 \mathbf{a}^{3}(0.780 \mathrm{~g}, 0.51 \mathrm{mmol})$ and toluene (15 $\mathrm{mL})$. Column chromatography $\left(1 \% \mathrm{NEt}_{3} / \mathrm{CH}_{2} \mathrm{Cl}_{2}\right)$ gave recovered $2(0.610 \mathrm{~g}, 98 \%)$ followed by ${ }_{\mathrm{p}} R-7 \mathbf{b}(0.561 \mathrm{~g}, 93 \%)$ as a bright orange-red foam.

${ }_{\mathrm{p}} \boldsymbol{R}-7 \mathbf{b}:[\alpha]_{\mathrm{D}}{ }^{25}+734\left(c 0.1, \mathrm{CH}_{2} \mathrm{Cl}_{2}\right)$.

Determination of the enantiomeric excess of $7 \mathbf{b}$. To a solution of $7 \mathbf{b}(0.005 \mathrm{~g}, 0.004 \mathrm{mmol})$ in $\mathrm{CDCl}_{3}(0.75 \mathrm{~mL})$ was added $(S)$ - $\alpha$-methylbenzylamine $(6 \mu \mathrm{L}, 0.05 \mathrm{mmol})$. After 5 minutes the resulting solution was examined by NMR spectroscopy.

${ }^{1} \mathrm{H}$ NMR: ${ }_{\mathrm{p}} S-7 \mathbf{b} 3.94,{ }_{\mathrm{p}} R-7 \mathbf{b} 3.97\left(5 \mathrm{H}, \mathrm{s}, \mathrm{C}_{5} \mathrm{H}_{5}\right) .{ }^{31} \mathrm{P}$ NMR: ${ }_{\mathrm{p}} S-7 \mathbf{b} 36.81,{ }_{\mathrm{p}} R-7 \mathbf{b} 36.90$. 
${ }^{1} \mathrm{H}$ NMR of ${ }_{\mathrm{p}} S-7 \mathbf{b}+(S)$ - $\alpha$-methylbenzylamine.

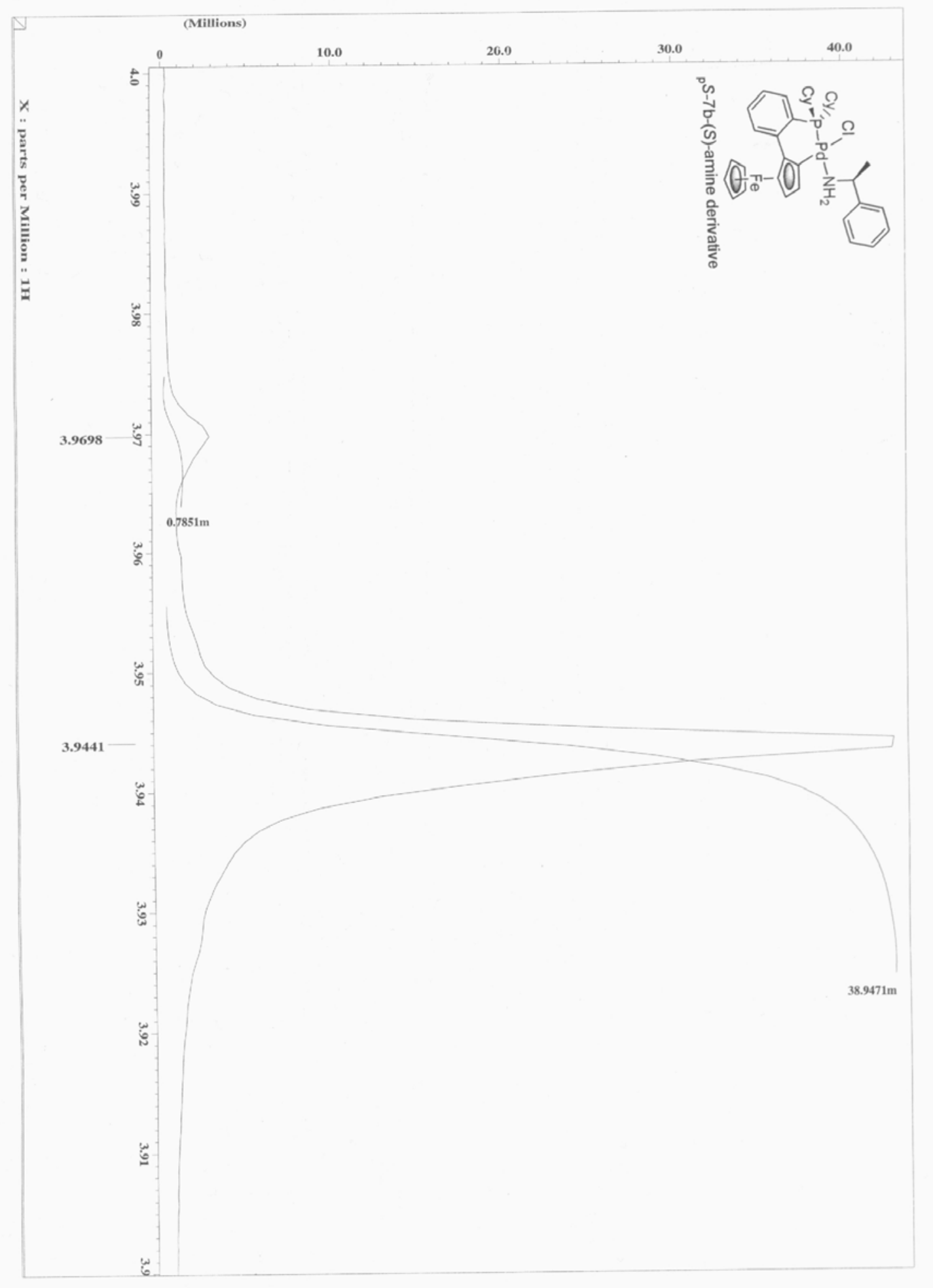


${ }^{1} \mathrm{H}$ NMR of ${ }_{\mathrm{p}} R-7 \mathbf{b}+(S)$ - $\alpha$-methylbenzylamine.

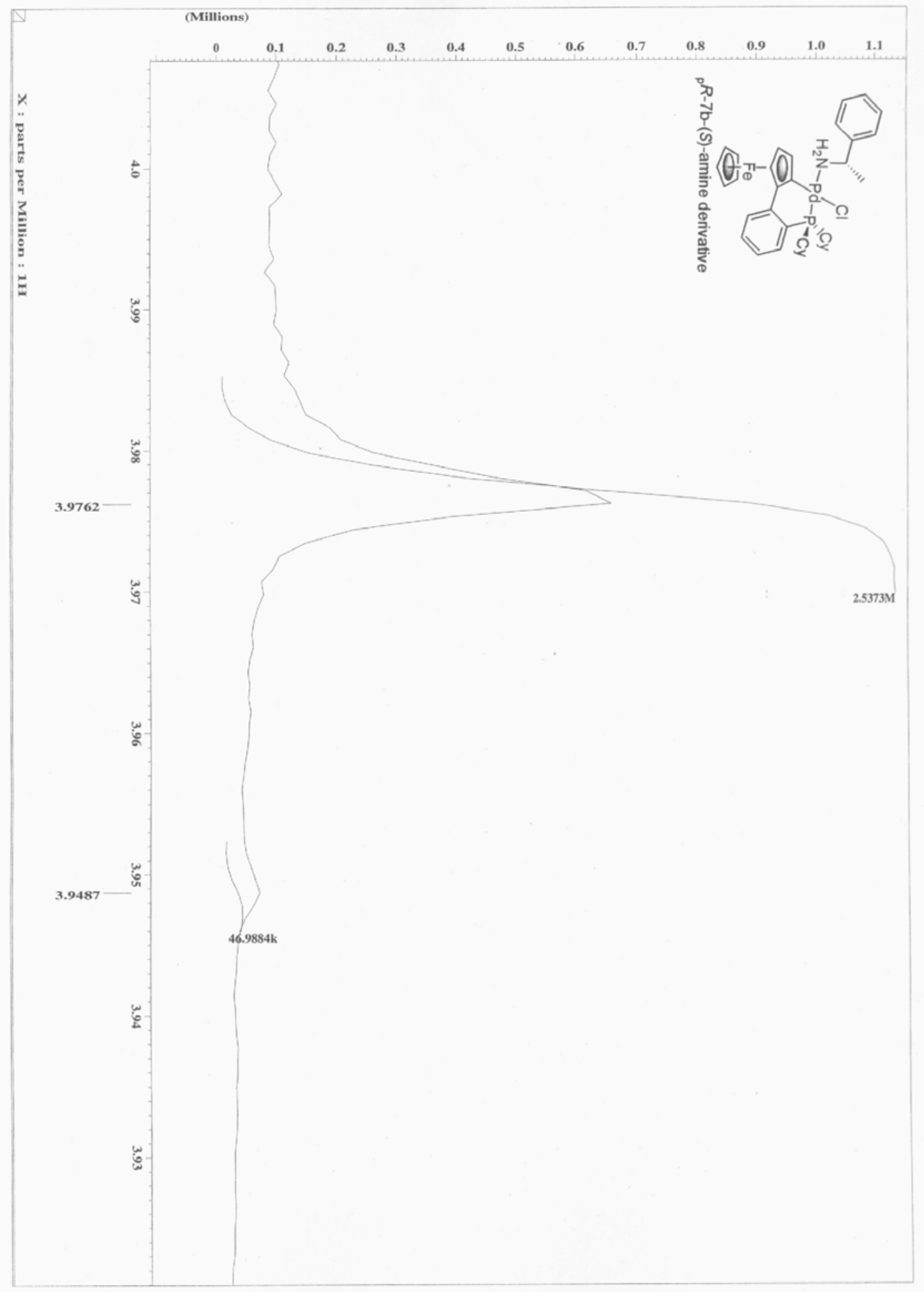


Synthesis of ${ }_{p} S$-di- $\mu$-chlorobis[2-(diphenylphosphino)phenylferrocene- $C^{1}, P$ dipalladium $8 b$.

The same procedure described for the synthesis of ${ }_{p} S-7 \mathbf{b}$ was employed using 2(diphenylphosphino)phenylferrocene $6(0.500 \mathrm{~g}, 1.12 \mathrm{mmol})$, di- $\mu$-acetatobis $\left[\left(\eta^{5}-(S)-\left({ }_{p} R\right)-2-\left(2^{\prime}-\right.\right.\right.$ (4'-methylethyl)oxazolinyl)cyclopentadienyl) $\left(\eta^{4}\right.$-tetraphenylcyclobutadiene)cobalt- $\left.C^{1}, N^{3}\right]$ dipalladium $3 \mathrm{a}^{2}(0.900 \mathrm{~g}, 0.60 \mathrm{mmol})$ and toluene $(35 \mathrm{~mL})$. Column chromatography $(1 \%$ $\left.\mathrm{NEt}_{3} / \mathrm{CH}_{2} \mathrm{Cl}_{2}\right)$ gave recovered $1(0.623 \mathrm{~g}, 88 \%)$ followed by ${ }_{\mathrm{p}} \mathrm{S}-\mathbf{8 b}(0.638 \mathrm{~g}, 97 \%)$ as a bright orange amorphous solid.

p S-8b: Anal. Found: C, 56.24; H, 4.25. calcd for $\mathrm{C}_{56} \mathrm{H}_{44} \mathrm{Cl}_{2} \mathrm{Fe}_{2} \mathrm{P}_{2} \mathrm{Pd}_{2} . \mathrm{H}_{2} \mathrm{O}$ : C, 56.41; H, 3.89. $[\alpha]_{\mathrm{D}}^{25}-480\left(c 0.1, \mathrm{CH}_{2} \mathrm{Cl}_{2}\right)$. Ratio of isomers $4: 1:{ }^{1} \mathrm{H}$ NMR $\left(\delta, 270 \mathrm{MHz}, \mathrm{CDCl}_{3}\right) 3.99$ (brs, $\mathrm{C}_{5} \mathrm{H}_{5}$ - minor) 4.08 (brs, $\mathrm{C}_{5} \mathrm{H}_{5}$ - major), 4.14 - 4.38 (2H, m, Fc), 4.60 (brs, Fc - major), 4.67 (brs, $\mathrm{Fc}$ - minor), $6.73(1 \mathrm{H}, \mathrm{t}, J 7, \mathrm{Ar}), 7.04(1 \mathrm{H}, \mathrm{t}, J 7, \mathrm{Ar}), 7.16-7.67(14 \mathrm{H}, \mathrm{m}, \mathrm{Ar}) .{ }^{13} \mathrm{C}\left\{{ }^{1} \mathrm{H}\right\} \mathrm{NMR}$ $\left(\delta, 67 \mathrm{MHz}, \mathrm{CDCl}_{3}\right)$ 67.34, 71.01, 71.36, 71.53, 76.19, 79.67, 78.00, 80.07, 80.36, 85.03, 86.35, $120.69,121.04,121.56,121.93,124.68,126.39,127.74-129.04,129.41,130.43-131.18,132.22-$ 132.39, 134.10-134.52, 135.72, 148.84, 148.92, 149.07, 149.12. ${ }^{31} \mathrm{P}\left\{{ }^{1} \mathrm{H}\right\} \operatorname{NMR}(\delta, 109 \mathrm{MHz}$, $\left.\mathrm{CDCl}_{3}\right) 31.74$ - minor, 32.15 - major $\left(\mathrm{PPh}_{2}\right)$, MS $\left(\mathrm{m} / \mathrm{z}\right.$, ES) $1174\left(\mathrm{M}^{+}-\mathrm{H}, 1 \%\right), 1137(\mathrm{M}-\mathrm{Cl}, 2)$, $551(1 / 2 \mathrm{M}-\mathrm{Cl}, 40 \%) .445(100)$.

\section{Synthesis of ${ }_{\mathrm{p}} R$-di- $\mu$-chlorobis[2-(diphenylphosphino)-phenylferrocene- $C^{1}, P$ ]dipalladium}

8b. The same procedure described for the synthesis of ${ }_{p} S-7 \mathbf{b}$ was employed using 2(diphenylphosphino)phenylferrocene $6(0.420 \mathrm{~g}, 0.94 \mathrm{mmol})$, di- $\mu$-acetatobis[ $\left[\eta^{5}-(S, \mathrm{p} S)-2-\left(2^{\prime}-\right.\right.$ (4'-tert-butyl)oxazolinyl)cyclopentadienyl)( $\eta^{4}$-tetraphenylcyclobutadiene)cobalt- $\left.C^{1}, N^{3}\right] \mathrm{di}$ palladium $4 \mathbf{a}^{3}(0.780 \mathrm{~g}, 0.51 \mathrm{mmol})$ and toluene $(15 \mathrm{~mL})$. Column chromatography $(1 \%$ $\left.\mathrm{NEt}_{3} / \mathrm{CH}_{2} \mathrm{Cl}_{2}\right)$ gave recovered $2(0.535 \mathrm{~g}, 88 \%)$ followed by ${ }_{\mathrm{p}} R-\mathbf{8 b}(0.494 \mathrm{~g}, 89 \%)$ as a dark orange amorphous solid.

${ }_{\mathbf{p}} \boldsymbol{R}-\mathbf{8 b}:[\alpha]_{\mathrm{D}}{ }^{25}+557\left(c 0.1, \mathrm{CH}_{2} \mathrm{Cl}_{2}\right)$.

Determination of the enantiomeric excess of $8 \mathbf{b}$. To a solution of $\mathbf{8 b}(0.005 \mathrm{~g}, 0.004 \mathrm{mmol})$ in $\mathrm{CDCl}_{3}(0.75 \mathrm{~mL})$ was added $(S)$-1-(1-naphthyl)ethylamine $(7 \mu \mathrm{L}, 0.04 \mathrm{mmol})$. After 5 minutes the resulting solution was examined by NMR spectroscopy.

${ }^{1} \mathrm{H}$ NMR: ${ }_{\mathrm{p}} S-7 \mathbf{b} 3.92,{ }_{\mathrm{p}} R-7 \mathbf{b} 3.86\left(5 \mathrm{H}, \mathrm{s}, \mathrm{C}_{5} \mathrm{H}_{5}\right)$. 
${ }^{1} \mathrm{H}$ NMR of ${ }_{\mathrm{p}} S-\mathbf{8 b}+(S)$-1-(1-naphthyl)ethylamine.

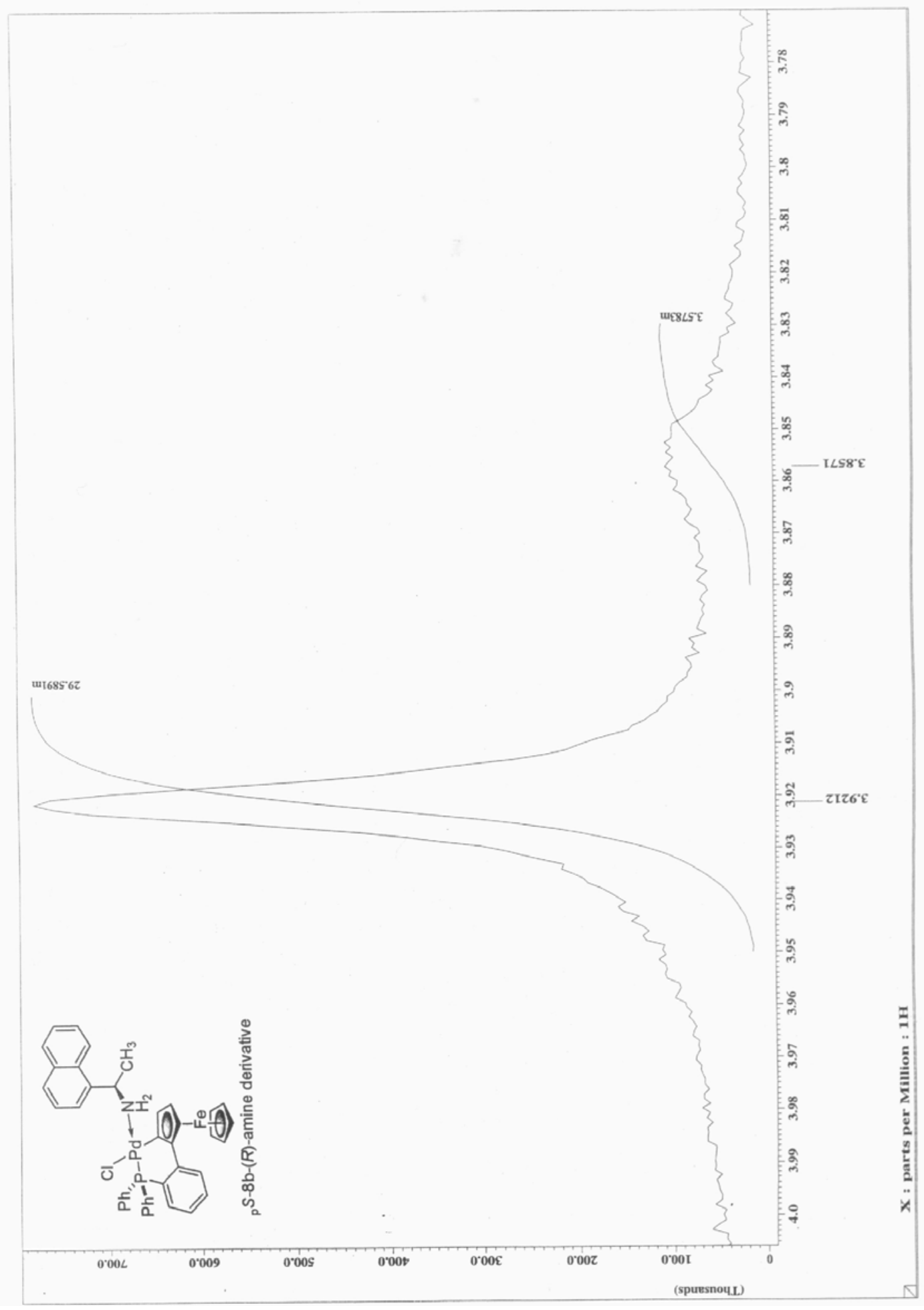


${ }^{1} \mathrm{H}$ NMR of ${ }_{\mathrm{p}} S-\mathbf{8 b}+(S)-1-(1-$ naphthyl)ethylamine.

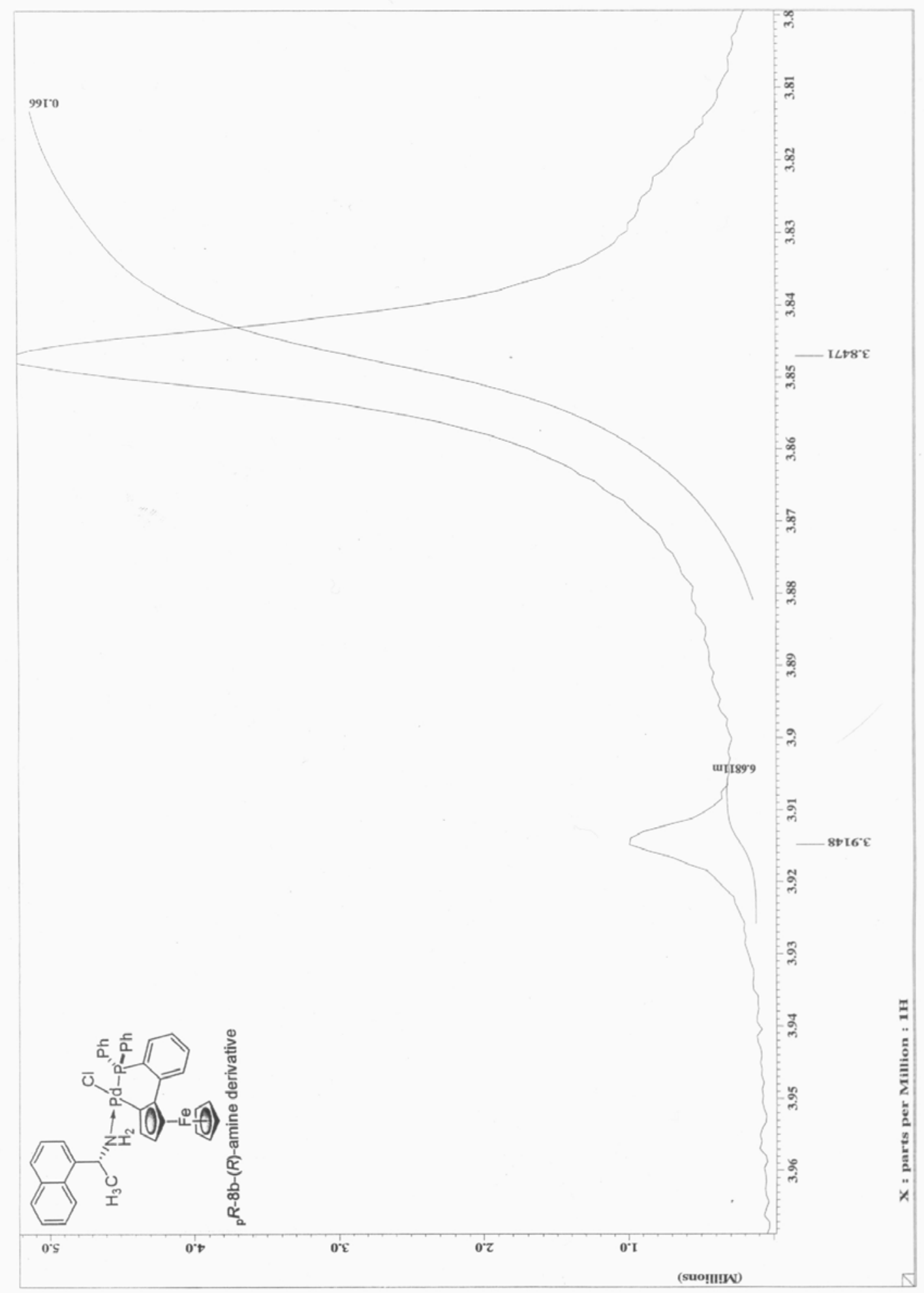


Isolation of 9b. To a stirred solution of di- $\mu$-chlorobis $\left[\left(\eta^{5}-(S)-\left({ }_{p} R\right)-2-\left(2^{\prime}-\left(4^{\prime}-\right.\right.\right.\right.$ methylethyl)oxazolinyl)cyclopentadienyl)( $\eta^{4}$-tetraphenylcyclobutadiene)cobalt$C^{1}, N^{3^{\prime}}$ ]dipalladium $3 \mathbf{b}(0.050 \mathrm{~g}, \quad 0.034 \mathrm{mmol})$ in toluene $(0.7 \mathrm{~mL})$ was added 2(dicyclohexylphosphino)phenylferrocene $5(0.031 \mathrm{~g}, 0.07 \mathrm{mmol})$ in one portion. After stirring for five minutes the solvent was removed in vacuo to give crude adduct $\mathbf{9 b}$.

${ }^{1} \mathrm{H}$ NMR $\left(\delta, 270 \mathrm{MHz}, \mathrm{CDCl}_{3}\right) 0.77\left(6 \mathrm{H}, \mathrm{d}, \mathrm{J} 7, \mathrm{CH}\left(\mathrm{CH}_{3}\right)_{2}\right), 0.8-2.0(23 \mathrm{H}, \mathrm{brm}, 2 \mathrm{x} \mathrm{Cy}+$ $\left.\mathrm{CH}\left(\mathrm{CH}_{3}\right)_{2}\right), 3.00(1 \mathrm{H}, \mathrm{brm}), 3.32(1 \mathrm{H}, \mathrm{brm}), 3.78(1 \mathrm{H}, \mathrm{brm}), 4.19(10 \mathrm{H}, \mathrm{brs}, \mathrm{Fc}+\mathrm{Cp}), 4.47(1 \mathrm{H}$, brs, Cp), 4.63 (1H, brs, Cp), 7.1 - 7.7 (23H, brm, Ar), 8.16 (1H, brs, Ar).

Crude 9a was similarly prepared. Mass spectra of $\mathbf{9 a} / \mathbf{b}$ below. 
Mass spectrum of 9a. Isotopic match [M-OAc $]^{+}$

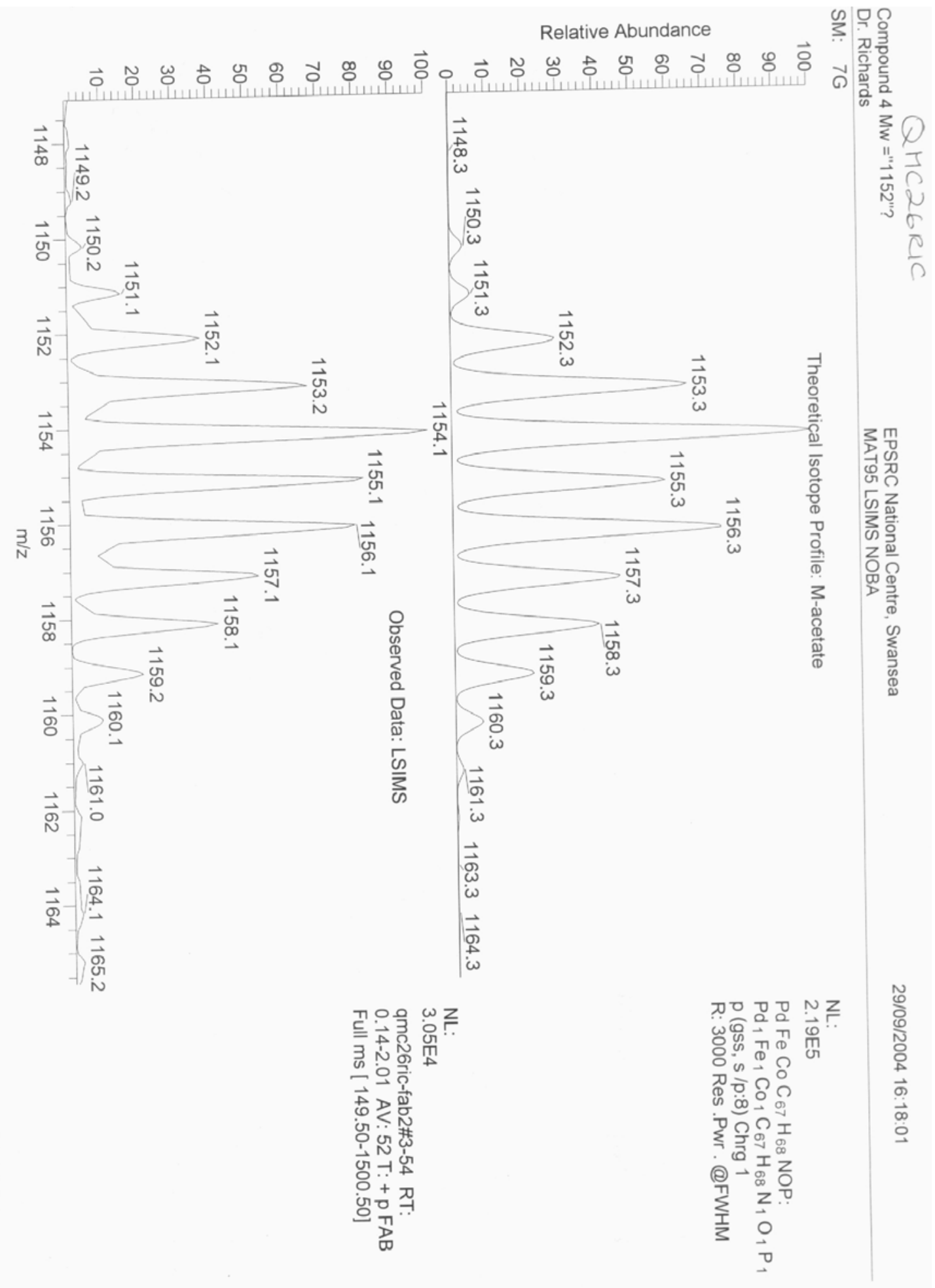


Mass spectrum of $\mathbf{9 b}$. Isotopic match $[\mathrm{M}]^{+}$

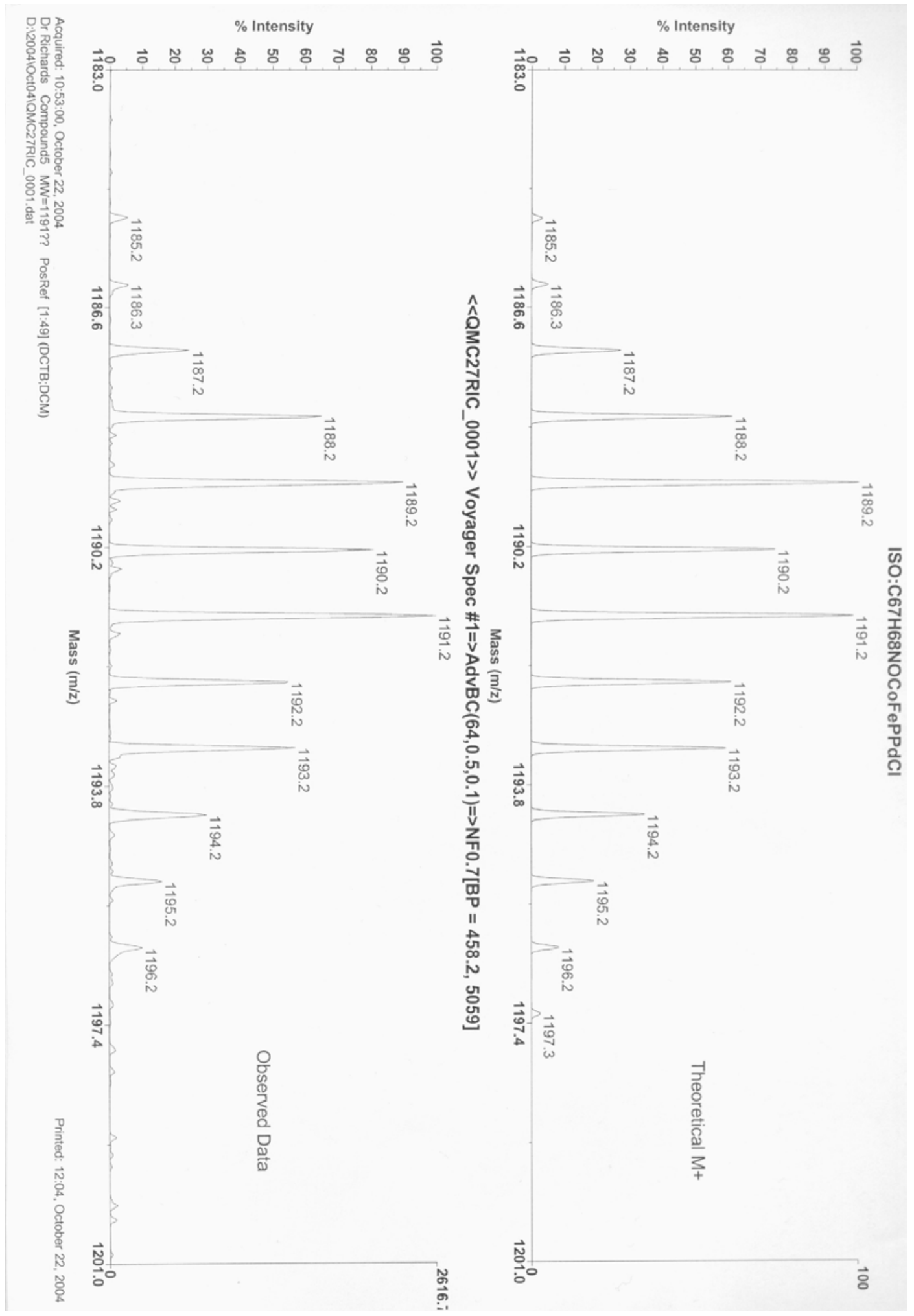

S12 


\section{X-Ray crystallography with $7 \mathrm{~b}$.}

Data were collected at $120 \mathrm{~K}$ using a Nonius Kappa CCD area detector diffractometer mounted at the window of molybdenum rotating anode $(50 \mathrm{KV}, 90 \mathrm{~mA}, \lambda=0.71069 \AA)$. The crystal-todetector distance was $30 \mathrm{~mm}$ and $\phi$ and $\Omega$ scans (2.0 increments, $12 \mathrm{~s}$ exposure time) were carried out to fill the Ewald sphere. Data collection and processing were carried out using the COLLECT $^{4}$, DENZO $^{5}$ and maXus ${ }^{6}$ and empirical absorption correction was applied using SADABS $^{7}$ The structure was solved by heavy- atom method using DIRDIF99 ${ }^{8}$ and refined anisotropically, (non-hydrogen atoms) by full-matrix least-squares on $F^{2}$ using the SHELXL-979 program. The $\mathrm{H}$ atoms were calculated geometrically and refined with riding model. The programs ORTEP- $3^{10}$ and PLATON ${ }^{11}$ were used for drawing the molecule. WINGX ${ }^{12}$ was used to prepare material for publication.

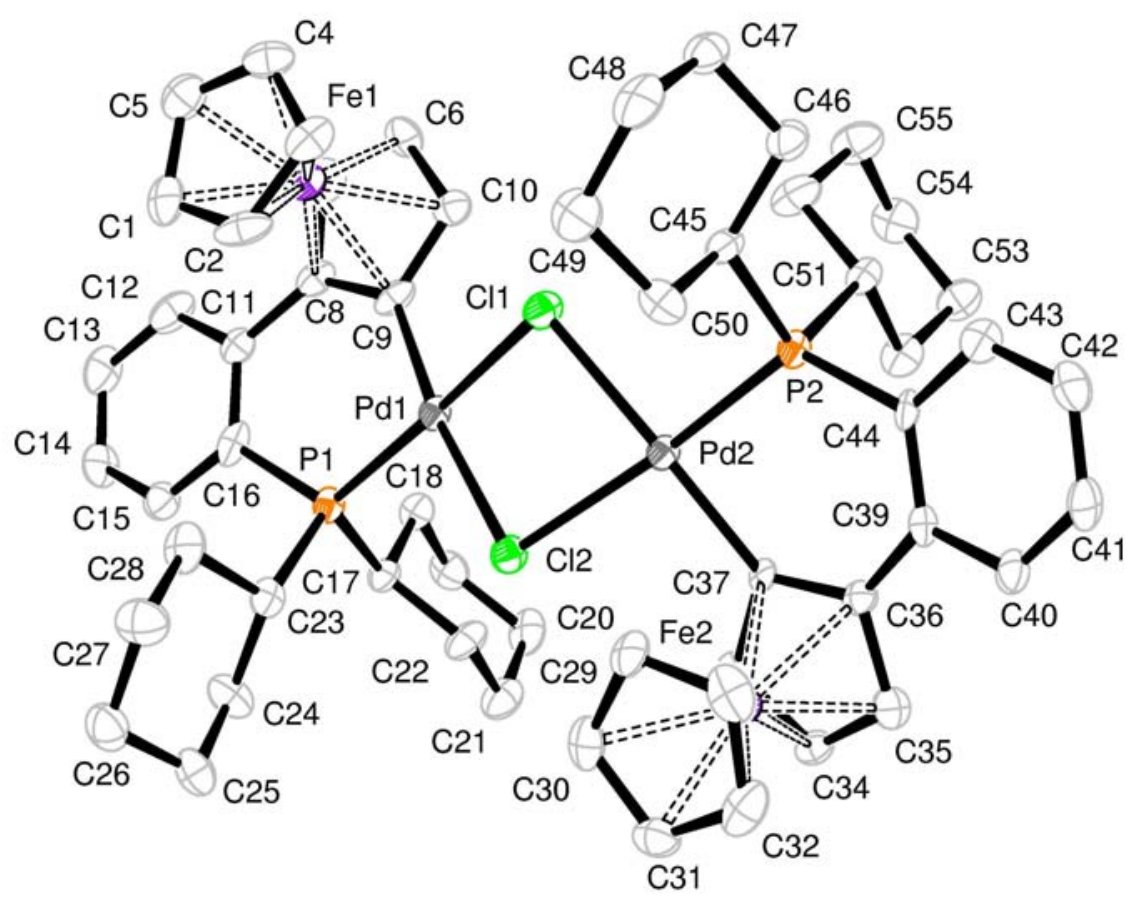

Representation of $\mathbf{7 b}$ [solvent of crystallisation $\left(2 \mathrm{x} \mathrm{CHCl}_{3}\right)$ omitted for clarity]. 
Table 1. Crystal data and structure refinement for $\mathbf{7 b}$.

Identification code

Empirical formula

Formula weight

Temperature

Wavelength

Crystal system

Space group

Unit cell dimensions

Volume

Z

Density (calculated)

Absorption coefficient

$\mathrm{F}(000)$

Crystal size

Theta range for data collection

Index ranges

Reflections collected

Independent reflections

Completeness to theta $=27.56^{\circ}$

Max. and min. transmission

Refinement method

Data / restraints / parameters

Goodness-of-fit on $\mathrm{F}^{2}$

Final R indices [I $>2 \operatorname{sigma}(\mathrm{I})]$

$\mathrm{R}$ indices (all data)

Absolute structure parameter

Largest diff. peak and hole xr-452-1c

$\mathrm{C}_{58} \mathrm{H}_{70} \mathrm{C}_{18} \mathrm{Fe}_{2} \mathrm{P}_{2} \mathrm{Pd}_{2}$

1437.18

120(2) K

$0.71073 \AA$

Monoclinic

$\mathrm{P} 2{ }_{1}$

$\begin{array}{ll}\mathrm{a}=13.5209(6) \AA & \alpha=90^{\circ} . \\ \mathrm{b}=15.1921(7) \AA & \beta=108.483(3)^{\circ} . \\ \mathrm{c}=15.0232(6) \AA & \gamma=90^{\circ} .\end{array}$

2926.7(2) $\AA^{3}$

2

$1.631 \mathrm{Mg} / \mathrm{m}^{3}$

$1.548 \mathrm{~mm}^{-1}$

1456

$0.08 \times 0.06 \times 0.02 \mathrm{~mm}^{3}$

3.33 to $27.56^{\circ}$.

$-17<=\mathrm{h}<=17,-19<=\mathrm{k}<=19,-19<=\mathrm{l}<=19$

36676

$13167[\mathrm{R}(\mathrm{int})=0.0776]$

$99.1 \%$

0.9697 and 0.8862

Full-matrix least-squares on $\mathrm{F}^{2}$

13167 / 1 / 649

1.016

$\mathrm{R} 1=0.0594, \mathrm{wR} 2=0.1068$

$\mathrm{R} 1=0.0855, \mathrm{wR} 2=0.1150$

$0.03(2)$

1.097 and -0.744 e. $\AA^{-3}$ 
Table 2. Atomic coordinates ( x 104) and equivalent isotropic displacement parameters $\left(\AA^{2} \times 10^{3}\right)$ for $\mathrm{xr}-452-1 \mathrm{c}$. $\mathrm{U}(\mathrm{eq})$ is defined as one third of the trace of the orthogonalized $U^{\mathrm{ij}}$ tensor.

\begin{tabular}{|c|c|c|c|c|}
\hline & $\mathrm{x}$ & $\mathrm{y}$ & $\mathrm{z}$ & $\mathrm{U}(\mathrm{eq})$ \\
\hline$C(1)$ & $4077(6)$ & $8506(5)$ & $6552(5)$ & $33(2)$ \\
\hline$C(2)$ & $3720(6)$ & $8958(5)$ & $5691(5)$ & $34(2)$ \\
\hline$C(3)$ & $2751(6)$ & $9378(5)$ & $5617(5)$ & $31(2)$ \\
\hline$C(4)$ & $2530(6)$ & $9169(5)$ & $6451(5)$ & $33(2)$ \\
\hline$C(5)$ & $3324(6)$ & $8652(5)$ & $7001(6)$ & $35(2)$ \\
\hline$C(6)$ & $1297(5)$ & $7347(5)$ & $5495(5)$ & $27(2)$ \\
\hline$C(7)$ & $2153(6)$ & $6841(5)$ & $5981(6)$ & $31(2)$ \\
\hline $\mathrm{C}(8)$ & $2852(6)$ & $6771(4)$ & $5443(5)$ & $23(2)$ \\
\hline$C(9)$ & $2422(6)$ & $7272(4)$ & $4601(5)$ & $24(2)$ \\
\hline$C(10)$ & $1442(5)$ & $7620(5)$ & $4625(5)$ & $25(2)$ \\
\hline $\mathrm{C}(11)$ & $3865(5)$ & $6280(4)$ & $5753(5)$ & $23(2)$ \\
\hline$C(12)$ & $4136(7)$ & $5839(5)$ & $6606(5)$ & $35(2)$ \\
\hline$C(13)$ & $5044(7)$ & $5324(5)$ & $6925(6)$ & $37(2)$ \\
\hline$C(14)$ & $5668(6)$ & $5245(5)$ & $6347(5)$ & $31(2)$ \\
\hline$C(15)$ & $5426(6)$ & $5690(5)$ & $5521(5)$ & $28(2)$ \\
\hline$C(16)$ & $4524(6)$ & $6215(4)$ & $5195(5)$ & $26(2)$ \\
\hline$C(17)$ & $3901(6)$ & $5574(4)$ & $3343(5)$ & $23(2)$ \\
\hline$C(18)$ & $3059(5)$ & $5041(5)$ & $3569(5)$ & $26(2)$ \\
\hline$C(19)$ & $2940(6)$ & $4141(5)$ & $3090(5)$ & $31(2)$ \\
\hline$C(20)$ & $2665(6)$ & $4234(5)$ & $2050(5)$ & $36(2)$ \\
\hline$C(21)$ & $3478(7)$ & $4798(5)$ & $1780(5)$ & $35(2)$ \\
\hline$C(22)$ & $3626(7)$ & $5689(5)$ & $2291(5)$ & $31(2)$ \\
\hline$C(23)$ & $5359(5)$ & $7104(4)$ & $3809(5)$ & $23(2)$ \\
\hline$C(24)$ & $6162(6)$ & $6465(5)$ & $3637(6)$ & $32(2)$ \\
\hline$C(25)$ & $6977(6)$ & $6999(5)$ & $3361(6)$ & $33(2)$ \\
\hline$C(26)$ & $7514(6)$ & $7635(6)$ & $4122(5)$ & $37(2)$ \\
\hline$C(27)$ & $6717(6)$ & $8272(6)$ & $4323(6)$ & $41(2)$ \\
\hline$C(28)$ & $5865(6)$ & $7771(5)$ & $4558(5)$ & $35(2)$ \\
\hline$C(29)$ & $3487(6)$ & $10105(5)$ & $505(5)$ & $26(2)$ \\
\hline$C(30)$ & $4140(5)$ & $9409(5)$ & $419(5)$ & $28(2)$ \\
\hline $\mathrm{C}(31)$ & $4129(6)$ & $9372(5)$ & $-519(5)$ & $29(2)$ \\
\hline
\end{tabular}




\begin{tabular}{|c|c|c|c|c|}
\hline$C(32)$ & $3477(6)$ & $10054(5)$ & $-999(5)$ & $34(2)$ \\
\hline$C(33)$ & $3069(6)$ & 10494(5) & $-384(6)$ & $32(2)$ \\
\hline $\mathrm{C}(34)$ & 1995(5) & $8073(4)$ & $-1117(4)$ & $21(1)$ \\
\hline$C(35)$ & $1369(5)$ & $8799(5)$ & $-1482(5)$ & $24(2)$ \\
\hline$C(36)$ & $1090(5)$ & $9217(4)$ & $-726(4)$ & $19(1)$ \\
\hline$C(37)$ & $1563(5)$ & $8739(4)$ & $106(4)$ & $15(1)$ \\
\hline $\mathrm{C}(38)$ & $2135(5)$ & $8021(4)$ & $-137(4)$ & $19(1)$ \\
\hline C(39) & $448(5)$ & $10014(4)$ & $-840(5)$ & $19(2)$ \\
\hline $\mathrm{C}(40)$ & $240(5)$ & $10512(5)$ & $-1683(5)$ & $24(2)$ \\
\hline $\mathrm{C}(41)$ & $-406(6)$ & $11223(5)$ & $-1852(5)$ & $27(2)$ \\
\hline$C(42)$ & $-893(6)$ & $11477(5)$ & $-1228(5)$ & $28(2)$ \\
\hline$C(43)$ & $-706(6)$ & 11007(4) & $-388(5)$ & $25(2)$ \\
\hline$C(44)$ & $-18(5)$ & $10299(4)$ & $-181(4)$ & $19(2)$ \\
\hline$C(45)$ & $540(5)$ & 10651(4) & 1861(5) & $22(2)$ \\
\hline$C(46)$ & $-320(6)$ & $11303(5)$ & $1880(5)$ & $28(2)$ \\
\hline$C(47)$ & $2(6)$ & $11827(5)$ & $2775(5)$ & $27(2)$ \\
\hline $\mathrm{C}(48)$ & 1011(6) & $12337(5)$ & $2862(5)$ & $35(2)$ \\
\hline$C(49)$ & $1889(6)$ & $11719(5)$ & $2815(6)$ & $39(2)$ \\
\hline$C(50)$ & $1542(6)$ & $11150(5)$ & 1915(6) & $30(2)$ \\
\hline$C(51)$ & $-973(5)$ & $9244(4)$ & 911(4) & $20(1)$ \\
\hline$C(52)$ & $-1133(6)$ & $8407(4)$ & $286(5)$ & $26(2)$ \\
\hline$C(53)$ & $-2190(6)$ & $7975(5)$ & $195(5)$ & $33(2)$ \\
\hline$C(54)$ & $-2283(6)$ & $7768(5)$ & $1147(5)$ & $37(2)$ \\
\hline$C(55)$ & $-2110(6)$ & $8595(5)$ & 1771(6) & $38(2)$ \\
\hline$C(56)$ & $-1050(6)$ & $9009(6)$ & $1871(5)$ & $36(2)$ \\
\hline$C(57)$ & $4574(6)$ & $6410(5)$ & $9613(6)$ & $37(2)$ \\
\hline $\mathrm{C}(58)$ & $560(7)$ & $5212(5)$ & $4737(5)$ & $36(2)$ \\
\hline $\mathrm{Cl}(1)$ & $1751(1)$ & $8896(1)$ & $3087(1)$ & $26(1)$ \\
\hline $\mathrm{Cl}(2)$ & $3305(1)$ & $8084(1)$ & 2113(1) & $23(1)$ \\
\hline $\mathrm{Cl}(3)$ & $4935(2)$ & $7074(2)$ & $8807(2)$ & $55(1)$ \\
\hline $\mathrm{Cl}(4)$ & 4664(2) & 6992(2) & $10632(2)$ & $47(1)$ \\
\hline $\mathrm{Cl}(5)$ & $3331(2)$ & $5963(2)$ & 9091(2) & $68(1)$ \\
\hline $\mathrm{Cl}(6)$ & $-529(2)$ & $5866(2)$ & $4222(2)$ & $54(1)$ \\
\hline $\mathrm{Cl}(7)$ & $627(2)$ & $4336(1)$ & $3988(1)$ & $39(1)$ \\
\hline $\mathrm{Cl}(8)$ & $564(3)$ & $4801(2)$ & $5814(2)$ & $90(1)$ \\
\hline $\mathrm{Fe}(1)$ & $2652(1)$ & $8048(1)$ & $5757(1)$ & $23(1)$ \\
\hline
\end{tabular}




$\begin{array}{lrrrr}\mathrm{Fe}(2) & 2659(1) & 9210(1) & -453(1) & 19(1) \\ \mathrm{P}(1) & 4166(1) & 6616(1) & 3995(1) & 20(1) \\ \mathrm{P}(2) & 279(1) & 9773(1) & 962(1) & 19(1) \\ \operatorname{Pd}(1) & 2922(1) & 7627(1) & 3537(1) & 19(1) \\ \operatorname{Pd}(2) & 1658(1) & 8894(1) & 1447(1) & 18(1)\end{array}$


Table 3. Bond lengths $[\AA]$ and angles $\left[^{\circ}\right]$ for $\mathrm{xr}-452-1 \mathrm{c}$.

\begin{tabular}{|c|c|}
\hline$C(1)-C(5)$ & $1.406(11)$ \\
\hline$C(1)-C(2)$ & $1.408(11)$ \\
\hline$C(1)-F e(1)$ & $2.041(7)$ \\
\hline $\mathrm{C}(1)-\mathrm{H}(1)$ & 0.9500 \\
\hline$C(2)-C(3)$ & $1.430(11)$ \\
\hline$C(2)-F e(1)$ & $2.024(7)$ \\
\hline $\mathrm{C}(2)-\mathrm{H}(2)$ & 0.9500 \\
\hline$C(3)-C(4)$ & $1.414(10)$ \\
\hline$C(3)-F e(1)$ & $2.041(7)$ \\
\hline $\mathrm{C}(3)-\mathrm{H}(3)$ & 0.9500 \\
\hline$C(4)-C(5)$ & $1.374(11)$ \\
\hline $\mathrm{C}(4)-\mathrm{Fe}(1)$ & $2.031(7)$ \\
\hline $\mathrm{C}(4)-\mathrm{H}(4)$ & 0.9500 \\
\hline$C(5)-F e(1)$ & $2.021(8)$ \\
\hline $\mathrm{C}(5)-\mathrm{H}(5)$ & 0.9500 \\
\hline$C(6)-C(7)$ & $1.388(10)$ \\
\hline$C(6)-C(10)$ & $1.442(10)$ \\
\hline$C(6)-F e(1)$ & $2.046(7)$ \\
\hline $\mathrm{C}(6)-\mathrm{H}(6)$ & 0.9500 \\
\hline$C(7)-C(8)$ & $1.429(10)$ \\
\hline $\mathrm{C}(7)-\mathrm{Fe}(1)$ & $2.019(7)$ \\
\hline $\mathrm{C}(7)-\mathrm{H}(7)$ & 0.9500 \\
\hline $\mathrm{C}(8)-\mathrm{C}(9)$ & $1.432(10)$ \\
\hline $\mathrm{C}(8)-\mathrm{C}(11)$ & $1.498(10)$ \\
\hline $\mathrm{C}(8)-\mathrm{Fe}(1)$ & $2.035(6)$ \\
\hline$C(9)-C(10)$ & $1.438(10)$ \\
\hline$C(9)-\operatorname{Pd}(1)$ & $1.998(7)$ \\
\hline$C(9)-F e(1)$ & $2.040(7)$ \\
\hline $\mathrm{C}(10)-\mathrm{Fe}(1)$ & $2.056(7)$ \\
\hline $\mathrm{C}(10)-\mathrm{H}(10)$ & 0.9500 \\
\hline $\mathrm{C}(11)-\mathrm{C}(12)$ & $1.388(10)$ \\
\hline$C(11)-C(16)$ & $1.408(10)$ \\
\hline $\mathrm{C}(12)-\mathrm{C}(13)$ & $1.406(11)$ \\
\hline $\mathrm{C}(12)-\mathrm{H}(12)$ & 0.9500 \\
\hline
\end{tabular}




\begin{tabular}{|c|c|}
\hline $\mathrm{C}(13)-\mathrm{C}(14)$ & $1.394(11)$ \\
\hline $\mathrm{C}(13)-\mathrm{H}(13)$ & 0.9500 \\
\hline $\mathrm{C}(14)-\mathrm{C}(15)$ & $1.359(10)$ \\
\hline $\mathrm{C}(14)-\mathrm{H}(14)$ & 0.9500 \\
\hline$C(15)-C(16)$ & $1.408(10)$ \\
\hline $\mathrm{C}(15)-\mathrm{H}(15)$ & 0.9500 \\
\hline $\mathrm{C}(16)-\mathrm{P}(1)$ & $1.816(7)$ \\
\hline $\mathrm{C}(17)-\mathrm{C}(22)$ & $1.514(9)$ \\
\hline $\mathrm{C}(17)-\mathrm{C}(18)$ & $1.522(10)$ \\
\hline $\mathrm{C}(17)-\mathrm{P}(1)$ & $1.836(7)$ \\
\hline $\mathrm{C}(17)-\mathrm{H}(17)$ & 1.0000 \\
\hline $\mathrm{C}(18)-\mathrm{C}(19)$ & $1.529(10)$ \\
\hline $\mathrm{C}(18)-\mathrm{H}(18 \mathrm{~A})$ & 0.9900 \\
\hline $\mathrm{C}(18)-\mathrm{H}(18 \mathrm{~B})$ & 0.9900 \\
\hline$C(19)-C(20)$ & $1.494(10)$ \\
\hline $\mathrm{C}(19)-\mathrm{H}(19 \mathrm{~A})$ & 0.9900 \\
\hline $\mathrm{C}(19)-\mathrm{H}(19 \mathrm{~B})$ & 0.9900 \\
\hline$C(20)-C(21)$ & $1.545(11)$ \\
\hline $\mathrm{C}(20)-\mathrm{H}(20 \mathrm{~A})$ & 0.9900 \\
\hline $\mathrm{C}(20)-\mathrm{H}(20 \mathrm{~B})$ & 0.9900 \\
\hline$C(21)-C(22)$ & $1.538(10)$ \\
\hline $\mathrm{C}(21)-\mathrm{H}(21 \mathrm{~A})$ & 0.9900 \\
\hline $\mathrm{C}(21)-\mathrm{H}(21 \mathrm{~B})$ & 0.9900 \\
\hline $\mathrm{C}(22)-\mathrm{H}(22 \mathrm{~A})$ & 0.9900 \\
\hline $\mathrm{C}(22)-\mathrm{H}(22 \mathrm{~B})$ & 0.9900 \\
\hline$C(23)-C(28)$ & $1.507(9)$ \\
\hline$C(23)-C(24)$ & $1.538(10)$ \\
\hline $\mathrm{C}(23)-\mathrm{P}(1)$ & $1.876(7)$ \\
\hline $\mathrm{C}(23)-\mathrm{H}(23)$ & 1.0000 \\
\hline$C(24)-C(25)$ & $1.528(10)$ \\
\hline $\mathrm{C}(24)-\mathrm{H}(24 \mathrm{~A})$ & 0.9900 \\
\hline $\mathrm{C}(24)-\mathrm{H}(24 \mathrm{~B})$ & 0.9900 \\
\hline$C(25)-C(26)$ & $1.498(11)$ \\
\hline $\mathrm{C}(25)-\mathrm{H}(25 \mathrm{~A})$ & 0.9900 \\
\hline $\mathrm{C}(25)-\mathrm{H}(25 \mathrm{~B})$ & 0.9900 \\
\hline$C(26)-C(27)$ & $1.547(11)$ \\
\hline
\end{tabular}




\begin{tabular}{|c|c|}
\hline $\mathrm{C}(26)-\mathrm{H}(26 \mathrm{~A})$ & 0.9900 \\
\hline $\mathrm{C}(26)-\mathrm{H}(26 \mathrm{~B})$ & 0.9900 \\
\hline $\mathrm{C}(27)-\mathrm{C}(28)$ & $1.513(10)$ \\
\hline $\mathrm{C}(27)-\mathrm{H}(27 \mathrm{~A})$ & 0.9900 \\
\hline $\mathrm{C}(27)-\mathrm{H}(27 \mathrm{~B})$ & 0.9900 \\
\hline $\mathrm{C}(28)-\mathrm{H}(28 \mathrm{~A})$ & 0.9900 \\
\hline $\mathrm{C}(28)-\mathrm{H}(28 \mathrm{~B})$ & 0.9900 \\
\hline C(29)-C(33) & $1.405(10)$ \\
\hline $\mathrm{C}(29)-\mathrm{C}(30)$ & $1.410(10)$ \\
\hline$C(29)-\mathrm{Fe}(2)$ & $2.038(7)$ \\
\hline C(29)-H(29) & 0.9500 \\
\hline $\mathrm{C}(30)-\mathrm{C}(31)$ & $1.404(10)$ \\
\hline $\mathrm{C}(30)-\mathrm{Fe}(2)$ & $2.038(7)$ \\
\hline $\mathrm{C}(30)-\mathrm{H}(30)$ & 0.9500 \\
\hline $\mathrm{C}(31)-\mathrm{C}(32)$ & $1.403(11)$ \\
\hline $\mathrm{C}(31)-\mathrm{Fe}(2)$ & $2.035(7)$ \\
\hline $\mathrm{C}(31)-\mathrm{H}(31)$ & 0.9500 \\
\hline $\mathrm{C}(32)-\mathrm{C}(33)$ & $1.387(11)$ \\
\hline $\mathrm{C}(32)-\mathrm{Fe}(2)$ & $2.028(8)$ \\
\hline $\mathrm{C}(32)-\mathrm{H}(32)$ & 0.9500 \\
\hline $\mathrm{C}(33)-\mathrm{Fe}(2)$ & $2.022(7)$ \\
\hline C(33)-H(33) & 0.9500 \\
\hline $\mathrm{C}(34)-\mathrm{C}(35)$ & $1.392(9)$ \\
\hline $\mathrm{C}(34)-\mathrm{C}(38)$ & $1.426(9)$ \\
\hline $\mathrm{C}(34)-\mathrm{Fe}(2)$ & $2.053(7)$ \\
\hline $\mathrm{C}(34)-\mathrm{H}(34)$ & 0.9500 \\
\hline $\mathrm{C}(35)-\mathrm{C}(36)$ & $1.453(9)$ \\
\hline $\mathrm{C}(35)-\mathrm{Fe}(2)$ & $2.028(7)$ \\
\hline $\mathrm{C}(35)-\mathrm{H}(35)$ & 0.9500 \\
\hline $\mathrm{C}(36)-\mathrm{C}(37)$ & $1.411(9)$ \\
\hline $\mathrm{C}(36)-\mathrm{C}(39)$ & $1.467(9)$ \\
\hline $\mathrm{C}(36)-\mathrm{Fe}(2)$ & $2.030(6)$ \\
\hline $\mathrm{C}(37)-\mathrm{C}(38)$ & $1.448(9)$ \\
\hline$C(37)-\operatorname{Pd}(2)$ & $1.992(6)$ \\
\hline $\mathrm{C}(37)-\mathrm{Fe}(2)$ & $2.050(6)$ \\
\hline $\mathrm{C}(38)-\mathrm{Fe}(2)$ & $2.050(6)$ \\
\hline
\end{tabular}




\begin{tabular}{|c|c|}
\hline $\mathrm{C}(38)-\mathrm{H}(38)$ & 0.9500 \\
\hline$C(39)-C(44)$ & $1.400(9)$ \\
\hline$C(39)-C(40)$ & $1.424(10)$ \\
\hline $\mathrm{C}(40)-\mathrm{C}(41)$ & $1.361(10)$ \\
\hline $\mathrm{C}(40)-\mathrm{H}(40)$ & 0.9500 \\
\hline$C(41)-C(42)$ & $1.361(10)$ \\
\hline $\mathrm{C}(41)-\mathrm{H}(41)$ & 0.9500 \\
\hline$C(42)-C(43)$ & $1.402(10)$ \\
\hline $\mathrm{C}(42)-\mathrm{H}(42)$ & 0.9500 \\
\hline $\mathrm{C}(43)-\mathrm{C}(44)$ & $1.390(9)$ \\
\hline $\mathrm{C}(43)-\mathrm{H}(43)$ & 0.9500 \\
\hline $\mathrm{C}(44)-\mathrm{P}(2)$ & $1.820(6)$ \\
\hline $\mathrm{C}(45)-\mathrm{C}(50)$ & $1.533(10)$ \\
\hline$C(45)-C(46)$ & $1.534(9)$ \\
\hline $\mathrm{C}(45)-\mathrm{P}(2)$ & $1.850(7)$ \\
\hline $\mathrm{C}(45)-\mathrm{H}(45)$ & 1.0000 \\
\hline $\mathrm{C}(46)-\mathrm{C}(47)$ & $1.503(10)$ \\
\hline $\mathrm{C}(46)-\mathrm{H}(46 \mathrm{~A})$ & 0.9900 \\
\hline $\mathrm{C}(46)-\mathrm{H}(46 \mathrm{~B})$ & 0.9900 \\
\hline $\mathrm{C}(47)-\mathrm{C}(48)$ & $1.538(11)$ \\
\hline $\mathrm{C}(47)-\mathrm{H}(47 \mathrm{~A})$ & 0.9900 \\
\hline $\mathrm{C}(47)-\mathrm{H}(47 \mathrm{~B})$ & 0.9900 \\
\hline $\mathrm{C}(48)-\mathrm{C}(49)$ & $1.533(11)$ \\
\hline $\mathrm{C}(48)-\mathrm{H}(48 \mathrm{~A})$ & 0.9900 \\
\hline $\mathrm{C}(48)-\mathrm{H}(48 \mathrm{~B})$ & 0.9900 \\
\hline$C(49)-C(50)$ & $1.547(10)$ \\
\hline $\mathrm{C}(49)-\mathrm{H}(49 \mathrm{~A})$ & 0.9900 \\
\hline $\mathrm{C}(49)-\mathrm{H}(49 \mathrm{~B})$ & 0.9900 \\
\hline $\mathrm{C}(50)-\mathrm{H}(50 \mathrm{~A})$ & 0.9900 \\
\hline $\mathrm{C}(50)-\mathrm{H}(50 \mathrm{~B})$ & 0.9900 \\
\hline $\mathrm{C}(51)-\mathrm{C}(56)$ & $1.521(9)$ \\
\hline $\mathrm{C}(51)-\mathrm{C}(52)$ & $1.554(9)$ \\
\hline $\mathrm{C}(51)-\mathrm{P}(2)$ & $1.854(7)$ \\
\hline $\mathrm{C}(51)-\mathrm{H}(51)$ & 1.0000 \\
\hline $\mathrm{C}(52)-\mathrm{C}(53)$ & $1.538(10)$ \\
\hline $\mathrm{C}(52)-\mathrm{H}(52 \mathrm{~A})$ & 0.9900 \\
\hline
\end{tabular}




\begin{tabular}{|c|c|}
\hline $\mathrm{C}(52)-\mathrm{H}(52 \mathrm{~B})$ & 0.9900 \\
\hline $\mathrm{C}(53)-\mathrm{C}(54)$ & $1.508(10)$ \\
\hline $\mathrm{C}(53)-\mathrm{H}(53 \mathrm{~A})$ & 0.9900 \\
\hline $\mathrm{C}(53)-\mathrm{H}(53 \mathrm{~B})$ & 0.9900 \\
\hline $\mathrm{C}(54)-\mathrm{C}(55)$ & $1.540(11)$ \\
\hline $\mathrm{C}(54)-\mathrm{H}(54 \mathrm{~A})$ & 0.9900 \\
\hline $\mathrm{C}(54)-\mathrm{H}(54 \mathrm{~B})$ & 0.9900 \\
\hline$C(55)-C(56)$ & $1.529(10)$ \\
\hline $\mathrm{C}(55)-\mathrm{H}(55 \mathrm{~A})$ & 0.9900 \\
\hline $\mathrm{C}(55)-\mathrm{H}(55 \mathrm{~B})$ & 0.9900 \\
\hline $\mathrm{C}(56)-\mathrm{H}(56 \mathrm{~A})$ & 0.9900 \\
\hline $\mathrm{C}(56)-\mathrm{H}(56 \mathrm{~B})$ & 0.9900 \\
\hline $\mathrm{C}(57)-\mathrm{Cl}(4)$ & $1.739(8)$ \\
\hline $\mathrm{C}(57)-\mathrm{Cl}(5)$ & $1.751(8)$ \\
\hline $\mathrm{C}(57)-\mathrm{Cl}(3)$ & $1.761(8)$ \\
\hline $\mathrm{C}(57)-\mathrm{H}(57)$ & 1.0000 \\
\hline $\mathrm{C}(58)-\mathrm{Cl}(8)$ & $1.734(8)$ \\
\hline $\mathrm{C}(58)-\mathrm{Cl}(6)$ & $1.742(8)$ \\
\hline $\mathrm{C}(58)-\mathrm{Cl}(7)$ & $1.763(8)$ \\
\hline $\mathrm{C}(58)-\mathrm{H}(58)$ & 1.0000 \\
\hline $\mathrm{Cl}(1)-\mathrm{Pd}(2)$ & $2.4267(16)$ \\
\hline $\mathrm{Cl}(1)-\mathrm{Pd}(1)$ & $2.4499(17)$ \\
\hline $\mathrm{Cl}(2)-\mathrm{Pd}(1)$ & $2.4542(17)$ \\
\hline $\mathrm{Cl}(2)-\mathrm{Pd}(2)$ & $2.4615(17)$ \\
\hline $\mathrm{P}(1)-\mathrm{Pd}(1)$ & $2.2196(18)$ \\
\hline $\mathrm{P}(2)-\mathrm{Pd}(2)$ & $2.2201(18)$ \\
\hline$C(5)-C(1)-C(2)$ & $105.9(7)$ \\
\hline $\mathrm{C}(5)-\mathrm{C}(1)-\mathrm{Fe}(1)$ & $69.0(4)$ \\
\hline $\mathrm{C}(2)-\mathrm{C}(1)-\mathrm{Fe}(1)$ & 69.1(4) \\
\hline $\mathrm{C}(5)-\mathrm{C}(1)-\mathrm{H}(1)$ & 127.0 \\
\hline $\mathrm{C}(2)-\mathrm{C}(1)-\mathrm{H}(1)$ & 127.0 \\
\hline $\mathrm{Fe}(1)-\mathrm{C}(1)-\mathrm{H}(1)$ & 126.4 \\
\hline$C(1)-C(2)-C(3)$ & $109.1(6)$ \\
\hline $\mathrm{C}(1)-\mathrm{C}(2)-\mathrm{Fe}(1)$ & $70.4(4)$ \\
\hline $\mathrm{C}(3)-\mathrm{C}(2)-\mathrm{Fe}(1)$ & $70.0(4)$ \\
\hline
\end{tabular}




\begin{tabular}{|c|c|}
\hline $\mathrm{C}(1)-\mathrm{C}(2)-\mathrm{H}(2)$ & 125.4 \\
\hline $\mathrm{C}(3)-\mathrm{C}(2)-\mathrm{H}(2)$ & 125.4 \\
\hline $\mathrm{Fe}(1)-\mathrm{C}(2)-\mathrm{H}(2)$ & 125.8 \\
\hline$C(4)-C(3)-C(2)$ & 106.1(7) \\
\hline $\mathrm{C}(4)-\mathrm{C}(3)-\mathrm{Fe}(1)$ & $69.3(4)$ \\
\hline$C(2)-C(3)-F e(1)$ & $68.8(4)$ \\
\hline $\mathrm{C}(4)-\mathrm{C}(3)-\mathrm{H}(3)$ & 127.0 \\
\hline $\mathrm{C}(2)-\mathrm{C}(3)-\mathrm{H}(3)$ & 127.0 \\
\hline $\mathrm{Fe}(1)-\mathrm{C}(3)-\mathrm{H}(3)$ & 126.5 \\
\hline$C(5)-C(4)-C(3)$ & $108.5(7)$ \\
\hline $\mathrm{C}(5)-\mathrm{C}(4)-\mathrm{Fe}(1)$ & $69.8(4)$ \\
\hline $\mathrm{C}(3)-\mathrm{C}(4)-\mathrm{Fe}(1)$ & $70.1(4)$ \\
\hline $\mathrm{C}(5)-\mathrm{C}(4)-\mathrm{H}(4)$ & 125.7 \\
\hline $\mathrm{C}(3)-\mathrm{C}(4)-\mathrm{H}(4)$ & 125.7 \\
\hline $\mathrm{Fe}(1)-\mathrm{C}(4)-\mathrm{H}(4)$ & 126.0 \\
\hline $\mathrm{C}(4)-\mathrm{C}(5)-\mathrm{C}(1)$ & $110.4(7)$ \\
\hline $\mathrm{C}(4)-\mathrm{C}(5)-\mathrm{Fe}(1)$ & $70.5(4)$ \\
\hline $\mathrm{C}(1)-\mathrm{C}(5)-\mathrm{Fe}(1)$ & $70.5(4)$ \\
\hline $\mathrm{C}(4)-\mathrm{C}(5)-\mathrm{H}(5)$ & 124.8 \\
\hline $\mathrm{C}(1)-\mathrm{C}(5)-\mathrm{H}(5)$ & 124.8 \\
\hline $\mathrm{Fe}(1)-\mathrm{C}(5)-\mathrm{H}(5)$ & 125.7 \\
\hline$C(7)-C(6)-C(10)$ & $107.9(7)$ \\
\hline $\mathrm{C}(7)-\mathrm{C}(6)-\mathrm{Fe}(1)$ & $69.0(4)$ \\
\hline $\mathrm{C}(10)-\mathrm{C}(6)-\mathrm{Fe}(1)$ & $69.8(4)$ \\
\hline $\mathrm{C}(7)-\mathrm{C}(6)-\mathrm{H}(6)$ & 126.1 \\
\hline $\mathrm{C}(10)-\mathrm{C}(6)-\mathrm{H}(6)$ & 126.1 \\
\hline $\mathrm{Fe}(1)-\mathrm{C}(6)-\mathrm{H}(6)$ & 126.7 \\
\hline$C(6)-C(7)-C(8)$ & $109.5(7)$ \\
\hline $\mathrm{C}(6)-\mathrm{C}(7)-\mathrm{Fe}(1)$ & $71.1(4)$ \\
\hline $\mathrm{C}(8)-\mathrm{C}(7)-\mathrm{Fe}(1)$ & $70.0(4)$ \\
\hline $\mathrm{C}(6)-\mathrm{C}(7)-\mathrm{H}(7)$ & 125.3 \\
\hline $\mathrm{C}(8)-\mathrm{C}(7)-\mathrm{H}(7)$ & 125.3 \\
\hline $\mathrm{Fe}(1)-\mathrm{C}(7)-\mathrm{H}(7)$ & 125.3 \\
\hline $\mathrm{C}(7)-\mathrm{C}(8)-\mathrm{C}(9)$ & $107.7(6)$ \\
\hline $\mathrm{C}(7)-\mathrm{C}(8)-\mathrm{C}(11)$ & $124.4(6)$ \\
\hline $\mathrm{C}(9)-\mathrm{C}(8)-\mathrm{C}(11)$ & $127.9(6)$ \\
\hline
\end{tabular}




\begin{tabular}{|c|c|}
\hline $\mathrm{C}(7)-\mathrm{C}(8)-\mathrm{Fe}(1)$ & $68.8(4)$ \\
\hline $\mathrm{C}(9)-\mathrm{C}(8)-\mathrm{Fe}(1)$ & $69.6(4)$ \\
\hline $\mathrm{C}(11)-\mathrm{C}(8)-\mathrm{Fe}(1)$ & $125.4(5)$ \\
\hline $\mathrm{C}(8)-\mathrm{C}(9)-\mathrm{C}(10)$ & 107.1(6) \\
\hline $\mathrm{C}(8)-\mathrm{C}(9)-\mathrm{Pd}(1)$ & $134.8(6)$ \\
\hline $\mathrm{C}(10)-\mathrm{C}(9)-\mathrm{Pd}(1)$ & $117.9(5)$ \\
\hline $\mathrm{C}(8)-\mathrm{C}(9)-\mathrm{Fe}(1)$ & $69.3(4)$ \\
\hline $\mathrm{C}(10)-\mathrm{C}(9)-\mathrm{Fe}(1)$ & $70.1(4)$ \\
\hline $\operatorname{Pd}(1)-C(9)-F e(1)$ & $121.8(3)$ \\
\hline $\mathrm{C}(9)-\mathrm{C}(10)-\mathrm{C}(6)$ & $107.8(6)$ \\
\hline $\mathrm{C}(9)-\mathrm{C}(10)-\mathrm{Fe}(1)$ & $68.8(4)$ \\
\hline $\mathrm{C}(6)-\mathrm{C}(10)-\mathrm{Fe}(1)$ & $69.0(4)$ \\
\hline $\mathrm{C}(9)-\mathrm{C}(10)-\mathrm{H}(10)$ & 126.1 \\
\hline $\mathrm{C}(6)-\mathrm{C}(10)-\mathrm{H}(10)$ & 126.1 \\
\hline $\mathrm{Fe}(1)-\mathrm{C}(10)-\mathrm{H}(10)$ & 127.6 \\
\hline $\mathrm{C}(12)-\mathrm{C}(11)-\mathrm{C}(16)$ & $119.0(7)$ \\
\hline $\mathrm{C}(12)-\mathrm{C}(11)-\mathrm{C}(8)$ & $118.3(7)$ \\
\hline$C(16)-C(11)-C(8)$ & $122.6(6)$ \\
\hline $\mathrm{C}(11)-\mathrm{C}(12)-\mathrm{C}(13)$ & $122.0(8)$ \\
\hline $\mathrm{C}(11)-\mathrm{C}(12)-\mathrm{H}(12)$ & 119.0 \\
\hline $\mathrm{C}(13)-\mathrm{C}(12)-\mathrm{H}(12)$ & 119.0 \\
\hline$C(14)-C(13)-C(12)$ & $118.1(7)$ \\
\hline $\mathrm{C}(14)-\mathrm{C}(13)-\mathrm{H}(13)$ & 121.0 \\
\hline $\mathrm{C}(12)-\mathrm{C}(13)-\mathrm{H}(13)$ & 121.0 \\
\hline $\mathrm{C}(15)-\mathrm{C}(14)-\mathrm{C}(13)$ & $120.5(7)$ \\
\hline $\mathrm{C}(15)-\mathrm{C}(14)-\mathrm{H}(14)$ & 119.7 \\
\hline $\mathrm{C}(13)-\mathrm{C}(14)-\mathrm{H}(14)$ & 119.7 \\
\hline$C(14)-C(15)-C(16)$ & $122.1(7)$ \\
\hline $\mathrm{C}(14)-\mathrm{C}(15)-\mathrm{H}(15)$ & 118.9 \\
\hline $\mathrm{C}(16)-\mathrm{C}(15)-\mathrm{H}(15)$ & 118.9 \\
\hline$C(15)-C(16)-C(11)$ & $118.3(6)$ \\
\hline $\mathrm{C}(15)-\mathrm{C}(16)-\mathrm{P}(1)$ & $117.7(6)$ \\
\hline $\mathrm{C}(11)-\mathrm{C}(16)-\mathrm{P}(1)$ & $123.2(5)$ \\
\hline$C(22)-C(17)-C(18)$ & $109.9(6)$ \\
\hline $\mathrm{C}(22)-\mathrm{C}(17)-\mathrm{P}(1)$ & $113.6(5)$ \\
\hline $\mathrm{C}(18)-\mathrm{C}(17)-\mathrm{P}(1)$ & $111.9(5)$ \\
\hline
\end{tabular}




\begin{tabular}{|c|c|}
\hline $\mathrm{C}(22)-\mathrm{C}(17)-\mathrm{H}(17)$ & 107.0 \\
\hline $\mathrm{C}(18)-\mathrm{C}(17)-\mathrm{H}(17)$ & 107.0 \\
\hline $\mathrm{P}(1)-\mathrm{C}(17)-\mathrm{H}(17)$ & 107.0 \\
\hline$C(17)-C(18)-C(19)$ & $110.2(6)$ \\
\hline $\mathrm{C}(17)-\mathrm{C}(18)-\mathrm{H}(18 \mathrm{~A})$ & 109.6 \\
\hline $\mathrm{C}(19)-\mathrm{C}(18)-\mathrm{H}(18 \mathrm{~A})$ & 109.6 \\
\hline $\mathrm{C}(17)-\mathrm{C}(18)-\mathrm{H}(18 \mathrm{~B})$ & 109.6 \\
\hline $\mathrm{C}(19)-\mathrm{C}(18)-\mathrm{H}(18 \mathrm{~B})$ & 109.6 \\
\hline $\mathrm{H}(18 \mathrm{~A})-\mathrm{C}(18)-\mathrm{H}(18 \mathrm{~B})$ & 108.1 \\
\hline $\mathrm{C}(20)-\mathrm{C}(19)-\mathrm{C}(18)$ & 111.2(6) \\
\hline $\mathrm{C}(20)-\mathrm{C}(19)-\mathrm{H}(19 \mathrm{~A})$ & 109.4 \\
\hline $\mathrm{C}(18)-\mathrm{C}(19)-\mathrm{H}(19 \mathrm{~A})$ & 109.4 \\
\hline $\mathrm{C}(20)-\mathrm{C}(19)-\mathrm{H}(19 \mathrm{~B})$ & 109.4 \\
\hline $\mathrm{C}(18)-\mathrm{C}(19)-\mathrm{H}(19 \mathrm{~B})$ & 109.4 \\
\hline $\mathrm{H}(19 \mathrm{~A})-\mathrm{C}(19)-\mathrm{H}(19 \mathrm{~B})$ & 108.0 \\
\hline $\mathrm{C}(19)-\mathrm{C}(20)-\mathrm{C}(21)$ & $111.4(6)$ \\
\hline $\mathrm{C}(19)-\mathrm{C}(20)-\mathrm{H}(20 \mathrm{~A})$ & 109.3 \\
\hline $\mathrm{C}(21)-\mathrm{C}(20)-\mathrm{H}(20 \mathrm{~A})$ & 109.3 \\
\hline $\mathrm{C}(19)-\mathrm{C}(20)-\mathrm{H}(20 \mathrm{~B})$ & 109.3 \\
\hline $\mathrm{C}(21)-\mathrm{C}(20)-\mathrm{H}(20 \mathrm{~B})$ & 109.3 \\
\hline $\mathrm{H}(20 \mathrm{~A})-\mathrm{C}(20)-\mathrm{H}(20 \mathrm{~B})$ & 108.0 \\
\hline $\mathrm{C}(22)-\mathrm{C}(21)-\mathrm{C}(20)$ & $110.3(6)$ \\
\hline $\mathrm{C}(22)-\mathrm{C}(21)-\mathrm{H}(21 \mathrm{~A})$ & 109.6 \\
\hline $\mathrm{C}(20)-\mathrm{C}(21)-\mathrm{H}(21 \mathrm{~A})$ & 109.6 \\
\hline $\mathrm{C}(22)-\mathrm{C}(21)-\mathrm{H}(21 \mathrm{~B})$ & 109.6 \\
\hline $\mathrm{C}(20)-\mathrm{C}(21)-\mathrm{H}(21 \mathrm{~B})$ & 109.6 \\
\hline $\mathrm{H}(21 \mathrm{~A})-\mathrm{C}(21)-\mathrm{H}(21 \mathrm{~B})$ & 108.1 \\
\hline $\mathrm{C}(17)-\mathrm{C}(22)-\mathrm{C}(21)$ & $111.7(6)$ \\
\hline $\mathrm{C}(17)-\mathrm{C}(22)-\mathrm{H}(22 \mathrm{~A})$ & 109.3 \\
\hline $\mathrm{C}(21)-\mathrm{C}(22)-\mathrm{H}(22 \mathrm{~A})$ & 109.3 \\
\hline $\mathrm{C}(17)-\mathrm{C}(22)-\mathrm{H}(22 \mathrm{~B})$ & 109.3 \\
\hline $\mathrm{C}(21)-\mathrm{C}(22)-\mathrm{H}(22 \mathrm{~B})$ & 109.3 \\
\hline $\mathrm{H}(22 \mathrm{~A})-\mathrm{C}(22)-\mathrm{H}(22 \mathrm{~B})$ & 107.9 \\
\hline$C(28)-C(23)-C(24)$ & $111.9(6)$ \\
\hline $\mathrm{C}(28)-\mathrm{C}(23)-\mathrm{P}(1)$ & $111.3(5)$ \\
\hline $\mathrm{C}(24)-\mathrm{C}(23)-\mathrm{P}(1)$ & $117.5(5)$ \\
\hline
\end{tabular}




\begin{tabular}{|c|c|}
\hline $\mathrm{C}(28)-\mathrm{C}(23)-\mathrm{H}(23)$ & 105.0 \\
\hline $\mathrm{C}(24)-\mathrm{C}(23)-\mathrm{H}(23)$ & 105.0 \\
\hline $\mathrm{P}(1)-\mathrm{C}(23)-\mathrm{H}(23)$ & 105.0 \\
\hline$C(25)-C(24)-C(23)$ & $108.6(6)$ \\
\hline $\mathrm{C}(25)-\mathrm{C}(24)-\mathrm{H}(24 \mathrm{~A})$ & 110.0 \\
\hline $\mathrm{C}(23)-\mathrm{C}(24)-\mathrm{H}(24 \mathrm{~A})$ & 110.0 \\
\hline $\mathrm{C}(25)-\mathrm{C}(24)-\mathrm{H}(24 \mathrm{~B})$ & 110.0 \\
\hline $\mathrm{C}(23)-\mathrm{C}(24)-\mathrm{H}(24 \mathrm{~B})$ & 110.0 \\
\hline $\mathrm{H}(24 \mathrm{~A})-\mathrm{C}(24)-\mathrm{H}(24 \mathrm{~B})$ & 108.4 \\
\hline $\mathrm{C}(26)-\mathrm{C}(25)-\mathrm{C}(24)$ & $110.6(7)$ \\
\hline $\mathrm{C}(26)-\mathrm{C}(25)-\mathrm{H}(25 \mathrm{~A})$ & 109.5 \\
\hline $\mathrm{C}(24)-\mathrm{C}(25)-\mathrm{H}(25 \mathrm{~A})$ & 109.5 \\
\hline $\mathrm{C}(26)-\mathrm{C}(25)-\mathrm{H}(25 \mathrm{~B})$ & 109.5 \\
\hline $\mathrm{C}(24)-\mathrm{C}(25)-\mathrm{H}(25 \mathrm{~B})$ & 109.5 \\
\hline $\mathrm{H}(25 \mathrm{~A})-\mathrm{C}(25)-\mathrm{H}(25 \mathrm{~B})$ & 108.1 \\
\hline$C(25)-C(26)-C(27)$ & $110.7(6)$ \\
\hline $\mathrm{C}(25)-\mathrm{C}(26)-\mathrm{H}(26 \mathrm{~A})$ & 109.5 \\
\hline$C(27)-C(26)-H(26 A)$ & 109.5 \\
\hline $\mathrm{C}(25)-\mathrm{C}(26)-\mathrm{H}(26 \mathrm{~B})$ & 109.5 \\
\hline $\mathrm{C}(27)-\mathrm{C}(26)-\mathrm{H}(26 \mathrm{~B})$ & 109.5 \\
\hline $\mathrm{H}(26 \mathrm{~A})-\mathrm{C}(26)-\mathrm{H}(26 \mathrm{~B})$ & 108.1 \\
\hline $\mathrm{C}(28)-\mathrm{C}(27)-\mathrm{C}(26)$ & $111.1(7)$ \\
\hline $\mathrm{C}(28)-\mathrm{C}(27)-\mathrm{H}(27 \mathrm{~A})$ & 109.4 \\
\hline $\mathrm{C}(26)-\mathrm{C}(27)-\mathrm{H}(27 \mathrm{~A})$ & 109.4 \\
\hline $\mathrm{C}(28)-\mathrm{C}(27)-\mathrm{H}(27 \mathrm{~B})$ & 109.4 \\
\hline $\mathrm{C}(26)-\mathrm{C}(27)-\mathrm{H}(27 \mathrm{~B})$ & 109.4 \\
\hline $\mathrm{H}(27 \mathrm{~A})-\mathrm{C}(27)-\mathrm{H}(27 \mathrm{~B})$ & 108.0 \\
\hline $\mathrm{C}(23)-\mathrm{C}(28)-\mathrm{C}(27)$ & $111.3(6)$ \\
\hline $\mathrm{C}(23)-\mathrm{C}(28)-\mathrm{H}(28 \mathrm{~A})$ & 109.4 \\
\hline $\mathrm{C}(27)-\mathrm{C}(28)-\mathrm{H}(28 \mathrm{~A})$ & 109.4 \\
\hline $\mathrm{C}(23)-\mathrm{C}(28)-\mathrm{H}(28 \mathrm{~B})$ & 109.4 \\
\hline $\mathrm{C}(27)-\mathrm{C}(28)-\mathrm{H}(28 \mathrm{~B})$ & 109.4 \\
\hline $\mathrm{H}(28 \mathrm{~A})-\mathrm{C}(28)-\mathrm{H}(28 \mathrm{~B})$ & 108.0 \\
\hline$C(33)-C(29)-C(30)$ & $107.6(7)$ \\
\hline$C(33)-C(29)-F e(2)$ & $69.1(4)$ \\
\hline $\mathrm{C}(30)-\mathrm{C}(29)-\mathrm{Fe}(2)$ & $69.8(4)$ \\
\hline
\end{tabular}




\begin{tabular}{|c|c|}
\hline $\mathrm{C}(33)-\mathrm{C}(29)-\mathrm{H}(29)$ & 126.2 \\
\hline $\mathrm{C}(30)-\mathrm{C}(29)-\mathrm{H}(29)$ & 126.2 \\
\hline $\mathrm{Fe}(2)-\mathrm{C}(29)-\mathrm{H}(29)$ & 126.5 \\
\hline $\mathrm{C}(31)-\mathrm{C}(30)-\mathrm{C}(29)$ & $108.3(7)$ \\
\hline $\mathrm{C}(31)-\mathrm{C}(30)-\mathrm{Fe}(2)$ & $69.7(4)$ \\
\hline $\mathrm{C}(29)-\mathrm{C}(30)-\mathrm{Fe}(2)$ & $69.8(4)$ \\
\hline $\mathrm{C}(31)-\mathrm{C}(30)-\mathrm{H}(30)$ & 125.9 \\
\hline $\mathrm{C}(29)-\mathrm{C}(30)-\mathrm{H}(30)$ & 125.9 \\
\hline $\mathrm{Fe}(2)-\mathrm{C}(30)-\mathrm{H}(30)$ & 126.2 \\
\hline $\mathrm{C}(32)-\mathrm{C}(31)-\mathrm{C}(30)$ & $107.0(7)$ \\
\hline $\mathrm{C}(32)-\mathrm{C}(31)-\mathrm{Fe}(2)$ & $69.5(4)$ \\
\hline $\mathrm{C}(30)-\mathrm{C}(31)-\mathrm{Fe}(2)$ & $69.9(4)$ \\
\hline $\mathrm{C}(32)-\mathrm{C}(31)-\mathrm{H}(31)$ & 126.5 \\
\hline $\mathrm{C}(30)-\mathrm{C}(31)-\mathrm{H}(31)$ & 126.5 \\
\hline $\mathrm{Fe}(2)-\mathrm{C}(31)-\mathrm{H}(31)$ & 125.6 \\
\hline $\mathrm{C}(33)-\mathrm{C}(32)-\mathrm{C}(31)$ & $109.3(7)$ \\
\hline $\mathrm{C}(33)-\mathrm{C}(32)-\mathrm{Fe}(2)$ & $69.7(4)$ \\
\hline $\mathrm{C}(31)-\mathrm{C}(32)-\mathrm{Fe}(2)$ & $70.1(4)$ \\
\hline $\mathrm{C}(33)-\mathrm{C}(32)-\mathrm{H}(32)$ & 125.3 \\
\hline $\mathrm{C}(31)-\mathrm{C}(32)-\mathrm{H}(32)$ & 125.3 \\
\hline $\mathrm{Fe}(2)-\mathrm{C}(32)-\mathrm{H}(32)$ & 126.4 \\
\hline $\mathrm{C}(32)-\mathrm{C}(33)-\mathrm{C}(29)$ & $107.8(7)$ \\
\hline $\mathrm{C}(32)-\mathrm{C}(33)-\mathrm{Fe}(2)$ & $70.2(4)$ \\
\hline $\mathrm{C}(29)-\mathrm{C}(33)-\mathrm{Fe}(2)$ & $70.4(4)$ \\
\hline $\mathrm{C}(32)-\mathrm{C}(33)-\mathrm{H}(33)$ & 126.1 \\
\hline $\mathrm{C}(29)-\mathrm{C}(33)-\mathrm{H}(33)$ & 126.1 \\
\hline $\mathrm{Fe}(2)-\mathrm{C}(33)-\mathrm{H}(33)$ & 124.9 \\
\hline $\mathrm{C}(35)-\mathrm{C}(34)-\mathrm{C}(38)$ & $108.4(6)$ \\
\hline $\mathrm{C}(35)-\mathrm{C}(34)-\mathrm{Fe}(2)$ & $69.1(4)$ \\
\hline $\mathrm{C}(38)-\mathrm{C}(34)-\mathrm{Fe}(2)$ & $69.6(4)$ \\
\hline $\mathrm{C}(35)-\mathrm{C}(34)-\mathrm{H}(34)$ & 125.8 \\
\hline $\mathrm{C}(38)-\mathrm{C}(34)-\mathrm{H}(34)$ & 125.8 \\
\hline $\mathrm{Fe}(2)-\mathrm{C}(34)-\mathrm{H}(34)$ & 127.1 \\
\hline $\mathrm{C}(34)-\mathrm{C}(35)-\mathrm{C}(36)$ & $108.3(6)$ \\
\hline $\mathrm{C}(34)-\mathrm{C}(35)-\mathrm{Fe}(2)$ & $71.0(4)$ \\
\hline $\mathrm{C}(36)-\mathrm{C}(35)-\mathrm{Fe}(2)$ & $69.1(4)$ \\
\hline
\end{tabular}




\begin{tabular}{|c|c|}
\hline $\mathrm{C}(34)-\mathrm{C}(35)-\mathrm{H}(35)$ & 125.8 \\
\hline $\mathrm{C}(36)-\mathrm{C}(35)-\mathrm{H}(35)$ & 125.8 \\
\hline $\mathrm{Fe}(2)-\mathrm{C}(35)-\mathrm{H}(35)$ & 125.6 \\
\hline$C(37)-C(36)-C(35)$ & $108.0(6)$ \\
\hline$C(37)-C(36)-C(39)$ & $127.9(6)$ \\
\hline$C(35)-C(36)-C(39)$ & $124.1(6)$ \\
\hline $\mathrm{C}(37)-\mathrm{C}(36)-\mathrm{Fe}(2)$ & $70.5(4)$ \\
\hline $\mathrm{C}(35)-\mathrm{C}(36)-\mathrm{Fe}(2)$ & $68.9(4)$ \\
\hline $\mathrm{C}(39)-\mathrm{C}(36)-\mathrm{Fe}(2)$ & $124.7(5)$ \\
\hline $\mathrm{C}(36)-\mathrm{C}(37)-\mathrm{C}(38)$ & $107.1(5)$ \\
\hline $\mathrm{C}(36)-\mathrm{C}(37)-\mathrm{Pd}(2)$ & $134.4(5)$ \\
\hline $\mathrm{C}(38)-\mathrm{C}(37)-\mathrm{Pd}(2)$ & $118.4(4)$ \\
\hline $\mathrm{C}(36)-\mathrm{C}(37)-\mathrm{Fe}(2)$ & $69.0(4)$ \\
\hline $\mathrm{C}(38)-\mathrm{C}(37)-\mathrm{Fe}(2)$ & $69.3(3)$ \\
\hline $\operatorname{Pd}(2)-C(37)-F e(2)$ & $123.4(3)$ \\
\hline $\mathrm{C}(34)-\mathrm{C}(38)-\mathrm{C}(37)$ & $108.2(6)$ \\
\hline $\mathrm{C}(34)-\mathrm{C}(38)-\mathrm{Fe}(2)$ & $69.8(4)$ \\
\hline $\mathrm{C}(37)-\mathrm{C}(38)-\mathrm{Fe}(2)$ & $69.3(3)$ \\
\hline $\mathrm{C}(34)-\mathrm{C}(38)-\mathrm{H}(38)$ & 125.9 \\
\hline $\mathrm{C}(37)-\mathrm{C}(38)-\mathrm{H}(38)$ & 125.9 \\
\hline $\mathrm{Fe}(2)-\mathrm{C}(38)-\mathrm{H}(38)$ & 126.6 \\
\hline$C(44)-C(39)-C(40)$ & $117.3(6)$ \\
\hline $\mathrm{C}(44)-\mathrm{C}(39)-\mathrm{C}(36)$ & $123.7(6)$ \\
\hline$C(40)-C(39)-C(36)$ & $119.0(6)$ \\
\hline$C(41)-C(40)-C(39)$ & $121.4(7)$ \\
\hline $\mathrm{C}(41)-\mathrm{C}(40)-\mathrm{H}(40)$ & 119.3 \\
\hline $\mathrm{C}(39)-\mathrm{C}(40)-\mathrm{H}(40)$ & 119.3 \\
\hline $\mathrm{C}(42)-\mathrm{C}(41)-\mathrm{C}(40)$ & $121.2(7)$ \\
\hline $\mathrm{C}(42)-\mathrm{C}(41)-\mathrm{H}(41)$ & 119.4 \\
\hline $\mathrm{C}(40)-\mathrm{C}(41)-\mathrm{H}(41)$ & 119.4 \\
\hline$C(41)-C(42)-C(43)$ & $119.2(7)$ \\
\hline $\mathrm{C}(41)-\mathrm{C}(42)-\mathrm{H}(42)$ & 120.4 \\
\hline $\mathrm{C}(43)-\mathrm{C}(42)-\mathrm{H}(42)$ & 120.4 \\
\hline$C(44)-C(43)-C(42)$ & $120.8(7)$ \\
\hline $\mathrm{C}(44)-\mathrm{C}(43)-\mathrm{H}(43)$ & 119.6 \\
\hline $\mathrm{C}(42)-\mathrm{C}(43)-\mathrm{H}(43)$ & 119.6 \\
\hline
\end{tabular}




\begin{tabular}{|c|c|}
\hline$C(43)-C(44)-C(39)$ & $120.0(6)$ \\
\hline $\mathrm{C}(43)-\mathrm{C}(44)-\mathrm{P}(2)$ & $118.9(5)$ \\
\hline $\mathrm{C}(39)-\mathrm{C}(44)-\mathrm{P}(2)$ & $121.1(5)$ \\
\hline$C(50)-C(45)-C(46)$ & $110.0(6)$ \\
\hline $\mathrm{C}(50)-\mathrm{C}(45)-\mathrm{P}(2)$ & $110.8(5)$ \\
\hline $\mathrm{C}(46)-\mathrm{C}(45)-\mathrm{P}(2)$ & $120.6(5)$ \\
\hline $\mathrm{C}(50)-\mathrm{C}(45)-\mathrm{H}(45)$ & 104.6 \\
\hline $\mathrm{C}(46)-\mathrm{C}(45)-\mathrm{H}(45)$ & 104.6 \\
\hline $\mathrm{P}(2)-\mathrm{C}(45)-\mathrm{H}(45)$ & 104.6 \\
\hline $\mathrm{C}(47)-\mathrm{C}(46)-\mathrm{C}(45)$ & $110.7(6)$ \\
\hline $\mathrm{C}(47)-\mathrm{C}(46)-\mathrm{H}(46 \mathrm{~A})$ & 109.5 \\
\hline $\mathrm{C}(45)-\mathrm{C}(46)-\mathrm{H}(46 \mathrm{~A})$ & 109.5 \\
\hline $\mathrm{C}(47)-\mathrm{C}(46)-\mathrm{H}(46 \mathrm{~B})$ & 109.5 \\
\hline $\mathrm{C}(45)-\mathrm{C}(46)-\mathrm{H}(46 \mathrm{~B})$ & 109.5 \\
\hline $\mathrm{H}(46 \mathrm{~A})-\mathrm{C}(46)-\mathrm{H}(46 \mathrm{~B})$ & 108.1 \\
\hline $\mathrm{C}(46)-\mathrm{C}(47)-\mathrm{C}(48)$ & $109.9(6)$ \\
\hline $\mathrm{C}(46)-\mathrm{C}(47)-\mathrm{H}(47 \mathrm{~A})$ & 109.7 \\
\hline $\mathrm{C}(48)-\mathrm{C}(47)-\mathrm{H}(47 \mathrm{~A})$ & 109.7 \\
\hline $\mathrm{C}(46)-\mathrm{C}(47)-\mathrm{H}(47 \mathrm{~B})$ & 109.7 \\
\hline $\mathrm{C}(48)-\mathrm{C}(47)-\mathrm{H}(47 \mathrm{~B})$ & 109.7 \\
\hline $\mathrm{H}(47 \mathrm{~A})-\mathrm{C}(47)-\mathrm{H}(47 \mathrm{~B})$ & 108.2 \\
\hline $\mathrm{C}(49)-\mathrm{C}(48)-\mathrm{C}(47)$ & $111.5(6)$ \\
\hline $\mathrm{C}(49)-\mathrm{C}(48)-\mathrm{H}(48 \mathrm{~A})$ & 109.3 \\
\hline $\mathrm{C}(47)-\mathrm{C}(48)-\mathrm{H}(48 \mathrm{~A})$ & 109.3 \\
\hline $\mathrm{C}(49)-\mathrm{C}(48)-\mathrm{H}(48 \mathrm{~B})$ & 109.3 \\
\hline $\mathrm{C}(47)-\mathrm{C}(48)-\mathrm{H}(48 \mathrm{~B})$ & 109.3 \\
\hline $\mathrm{H}(48 \mathrm{~A})-\mathrm{C}(48)-\mathrm{H}(48 \mathrm{~B})$ & 108.0 \\
\hline$C(48)-C(49)-C(50)$ & $111.0(6)$ \\
\hline $\mathrm{C}(48)-\mathrm{C}(49)-\mathrm{H}(49 \mathrm{~A})$ & 109.4 \\
\hline $\mathrm{C}(50)-\mathrm{C}(49)-\mathrm{H}(49 \mathrm{~A})$ & 109.4 \\
\hline $\mathrm{C}(48)-\mathrm{C}(49)-\mathrm{H}(49 \mathrm{~B})$ & 109.4 \\
\hline $\mathrm{C}(50)-\mathrm{C}(49)-\mathrm{H}(49 \mathrm{~B})$ & 109.4 \\
\hline $\mathrm{H}(49 \mathrm{~A})-\mathrm{C}(49)-\mathrm{H}(49 \mathrm{~B})$ & 108.0 \\
\hline$C(45)-C(50)-C(49)$ & $109.9(6)$ \\
\hline $\mathrm{C}(45)-\mathrm{C}(50)-\mathrm{H}(50 \mathrm{~A})$ & 109.7 \\
\hline $\mathrm{C}(49)-\mathrm{C}(50)-\mathrm{H}(50 \mathrm{~A})$ & 109.7 \\
\hline
\end{tabular}




\begin{tabular}{|c|c|}
\hline $\mathrm{C}(45)-\mathrm{C}(50)-\mathrm{H}(50 \mathrm{~B})$ & 109.7 \\
\hline $\mathrm{C}(49)-\mathrm{C}(50)-\mathrm{H}(50 \mathrm{~B})$ & 109.7 \\
\hline $\mathrm{H}(50 \mathrm{~A})-\mathrm{C}(50)-\mathrm{H}(50 \mathrm{~B})$ & 108.2 \\
\hline$C(56)-C(51)-C(52)$ & $110.1(6)$ \\
\hline $\mathrm{C}(56)-\mathrm{C}(51)-\mathrm{P}(2)$ & $113.5(5)$ \\
\hline $\mathrm{C}(52)-\mathrm{C}(51)-\mathrm{P}(2)$ & $109.3(5)$ \\
\hline $\mathrm{C}(56)-\mathrm{C}(51)-\mathrm{H}(51)$ & 107.9 \\
\hline $\mathrm{C}(52)-\mathrm{C}(51)-\mathrm{H}(51)$ & 107.9 \\
\hline $\mathrm{P}(2)-\mathrm{C}(51)-\mathrm{H}(51)$ & 107.9 \\
\hline $\mathrm{C}(53)-\mathrm{C}(52)-\mathrm{C}(51)$ & $110.5(6)$ \\
\hline $\mathrm{C}(53)-\mathrm{C}(52)-\mathrm{H}(52 \mathrm{~A})$ & 109.6 \\
\hline $\mathrm{C}(51)-\mathrm{C}(52)-\mathrm{H}(52 \mathrm{~A})$ & 109.6 \\
\hline $\mathrm{C}(53)-\mathrm{C}(52)-\mathrm{H}(52 \mathrm{~B})$ & 109.6 \\
\hline $\mathrm{C}(51)-\mathrm{C}(52)-\mathrm{H}(52 \mathrm{~B})$ & 109.6 \\
\hline $\mathrm{H}(52 \mathrm{~A})-\mathrm{C}(52)-\mathrm{H}(52 \mathrm{~B})$ & 108.1 \\
\hline $\mathrm{C}(54)-\mathrm{C}(53)-\mathrm{C}(52)$ & $111.0(6)$ \\
\hline $\mathrm{C}(54)-\mathrm{C}(53)-\mathrm{H}(53 \mathrm{~A})$ & 109.4 \\
\hline $\mathrm{C}(52)-\mathrm{C}(53)-\mathrm{H}(53 \mathrm{~A})$ & 109.4 \\
\hline $\mathrm{C}(54)-\mathrm{C}(53)-\mathrm{H}(53 \mathrm{~B})$ & 109.4 \\
\hline $\mathrm{C}(52)-\mathrm{C}(53)-\mathrm{H}(53 \mathrm{~B})$ & 109.4 \\
\hline $\mathrm{H}(53 \mathrm{~A})-\mathrm{C}(53)-\mathrm{H}(53 \mathrm{~B})$ & 108.0 \\
\hline$C(53)-C(54)-C(55)$ & $111.4(6)$ \\
\hline $\mathrm{C}(53)-\mathrm{C}(54)-\mathrm{H}(54 \mathrm{~A})$ & 109.3 \\
\hline $\mathrm{C}(55)-\mathrm{C}(54)-\mathrm{H}(54 \mathrm{~A})$ & 109.3 \\
\hline $\mathrm{C}(53)-\mathrm{C}(54)-\mathrm{H}(54 \mathrm{~B})$ & 109.3 \\
\hline $\mathrm{C}(55)-\mathrm{C}(54)-\mathrm{H}(54 \mathrm{~B})$ & 109.3 \\
\hline $\mathrm{H}(54 \mathrm{~A})-\mathrm{C}(54)-\mathrm{H}(54 \mathrm{~B})$ & 108.0 \\
\hline $\mathrm{C}(56)-\mathrm{C}(55)-\mathrm{C}(54)$ & $110.6(6)$ \\
\hline $\mathrm{C}(56)-\mathrm{C}(55)-\mathrm{H}(55 \mathrm{~A})$ & 109.5 \\
\hline $\mathrm{C}(54)-\mathrm{C}(55)-\mathrm{H}(55 \mathrm{~A})$ & 109.5 \\
\hline $\mathrm{C}(56)-\mathrm{C}(55)-\mathrm{H}(55 \mathrm{~B})$ & 109.5 \\
\hline $\mathrm{C}(54)-\mathrm{C}(55)-\mathrm{H}(55 \mathrm{~B})$ & 109.5 \\
\hline $\mathrm{H}(55 \mathrm{~A})-\mathrm{C}(55)-\mathrm{H}(55 \mathrm{~B})$ & 108.1 \\
\hline$C(51)-C(56)-C(55)$ & $110.3(6)$ \\
\hline $\mathrm{C}(51)-\mathrm{C}(56)-\mathrm{H}(56 \mathrm{~A})$ & 109.6 \\
\hline $\mathrm{C}(55)-\mathrm{C}(56)-\mathrm{H}(56 \mathrm{~A})$ & 109.6 \\
\hline
\end{tabular}




\begin{tabular}{|c|c|}
\hline $\mathrm{C}(51)-\mathrm{C}(56)-\mathrm{H}(56 \mathrm{~B})$ & 109.6 \\
\hline $\mathrm{C}(55)-\mathrm{C}(56)-\mathrm{H}(56 \mathrm{~B})$ & 109.6 \\
\hline $\mathrm{H}(56 \mathrm{~A})-\mathrm{C}(56)-\mathrm{H}(56 \mathrm{~B})$ & 108.1 \\
\hline $\mathrm{Cl}(4)-\mathrm{C}(57)-\mathrm{Cl}(5)$ & $112.4(5)$ \\
\hline $\mathrm{Cl}(4)-\mathrm{C}(57)-\mathrm{Cl}(3)$ & $110.9(4)$ \\
\hline $\mathrm{Cl}(5)-\mathrm{C}(57)-\mathrm{Cl}(3)$ & $110.5(4)$ \\
\hline $\mathrm{Cl}(4)-\mathrm{C}(57)-\mathrm{H}(57)$ & 107.6 \\
\hline $\mathrm{Cl}(5)-\mathrm{C}(57)-\mathrm{H}(57)$ & 107.6 \\
\hline $\mathrm{Cl}(3)-\mathrm{C}(57)-\mathrm{H}(57)$ & 107.6 \\
\hline $\mathrm{Cl}(8)-\mathrm{C}(58)-\mathrm{Cl}(6)$ & $111.9(5)$ \\
\hline $\mathrm{Cl}(8)-\mathrm{C}(58)-\mathrm{Cl}(7)$ & $109.8(4)$ \\
\hline $\mathrm{Cl}(6)-\mathrm{C}(58)-\mathrm{Cl}(7)$ & $110.7(4)$ \\
\hline $\mathrm{Cl}(8)-\mathrm{C}(58)-\mathrm{H}(58)$ & 108.1 \\
\hline $\mathrm{Cl}(6)-\mathrm{C}(58)-\mathrm{H}(58)$ & 108.1 \\
\hline $\mathrm{Cl}(7)-\mathrm{C}(58)-\mathrm{H}(58)$ & 108.1 \\
\hline $\operatorname{Pd}(2)-\mathrm{Cl}(1)-\operatorname{Pd}(1)$ & $95.74(6)$ \\
\hline $\operatorname{Pd}(1)-\mathrm{Cl}(2)-\operatorname{Pd}(2)$ & $94.74(6)$ \\
\hline $\mathrm{C}(7)-\mathrm{Fe}(1)-\mathrm{C}(5)$ & 109.6(3) \\
\hline $\mathrm{C}(7)-\mathrm{Fe}(1)-\mathrm{C}(2)$ & $155.5(3)$ \\
\hline $\mathrm{C}(5)-\mathrm{Fe}(1)-\mathrm{C}(2)$ & $67.5(3)$ \\
\hline $\mathrm{C}(7)-\mathrm{Fe}(1)-\mathrm{C}(4)$ & $126.2(3)$ \\
\hline $\mathrm{C}(5)-\mathrm{Fe}(1)-\mathrm{C}(4)$ & $39.7(3)$ \\
\hline $\mathrm{C}(2)-\mathrm{Fe}(1)-\mathrm{C}(4)$ & $68.1(3)$ \\
\hline $\mathrm{C}(7)-\mathrm{Fe}(1)-\mathrm{C}(8)$ & $41.3(3)$ \\
\hline $\mathrm{C}(5)-\mathrm{Fe}(1)-\mathrm{C}(8)$ & $126.4(3)$ \\
\hline $\mathrm{C}(2)-\mathrm{Fe}(1)-\mathrm{C}(8)$ & 119.3(3) \\
\hline $\mathrm{C}(4)-\mathrm{Fe}(1)-\mathrm{C}(8)$ & $163.2(3)$ \\
\hline $\mathrm{C}(7)-\mathrm{Fe}(1)-\mathrm{C}(9)$ & $69.4(3)$ \\
\hline $\mathrm{C}(5)-\mathrm{Fe}(1)-\mathrm{C}(9)$ & $162.2(3)$ \\
\hline $\mathrm{C}(2)-\mathrm{Fe}(1)-\mathrm{C}(9)$ & $105.7(3)$ \\
\hline $\mathrm{C}(4)-\mathrm{Fe}(1)-\mathrm{C}(9)$ & 155.1(3) \\
\hline $\mathrm{C}(8)-\mathrm{Fe}(1)-\mathrm{C}(9)$ & $41.1(3)$ \\
\hline $\mathrm{C}(7)-\mathrm{Fe}(1)-\mathrm{C}(1)$ & $121.0(3)$ \\
\hline $\mathrm{C}(5)-\mathrm{Fe}(1)-\mathrm{C}(1)$ & $40.5(3)$ \\
\hline $\mathrm{C}(2)-\mathrm{Fe}(1)-\mathrm{C}(1)$ & $40.5(3)$ \\
\hline $\mathrm{C}(4)-\mathrm{Fe}(1)-\mathrm{C}(1)$ & $68.2(3)$ \\
\hline
\end{tabular}




\begin{tabular}{|c|c|}
\hline $\mathrm{C}(8)-\mathrm{Fe}(1)-\mathrm{C}(1)$ & $106.8(3)$ \\
\hline $\mathrm{C}(9)-\mathrm{Fe}(1)-\mathrm{C}(1)$ & $123.8(3)$ \\
\hline $\mathrm{C}(7)-\mathrm{Fe}(1)-\mathrm{C}(3)$ & $162.4(3)$ \\
\hline $\mathrm{C}(5)-\mathrm{Fe}(1)-\mathrm{C}(3)$ & $67.7(3)$ \\
\hline $\mathrm{C}(2)-\mathrm{Fe}(1)-\mathrm{C}(3)$ & $41.2(3)$ \\
\hline $\mathrm{C}(4)-\mathrm{Fe}(1)-\mathrm{C}(3)$ & $40.6(3)$ \\
\hline $\mathrm{C}(8)-\mathrm{Fe}(1)-\mathrm{C}(3)$ & $154.4(3)$ \\
\hline $\mathrm{C}(9)-\mathrm{Fe}(1)-\mathrm{C}(3)$ & $118.8(3)$ \\
\hline $\mathrm{C}(1)-\mathrm{Fe}(1)-\mathrm{C}(3)$ & $69.0(3)$ \\
\hline $\mathrm{C}(7)-\mathrm{Fe}(1)-\mathrm{C}(6)$ & $39.9(3)$ \\
\hline $\mathrm{C}(5)-\mathrm{Fe}(1)-\mathrm{C}(6)$ & $121.8(3)$ \\
\hline $\mathrm{C}(2)-\mathrm{Fe}(1)-\mathrm{C}(6)$ & $162.8(3)$ \\
\hline $\mathrm{C}(4)-\mathrm{Fe}(1)-\mathrm{C}(6)$ & $108.8(3)$ \\
\hline $\mathrm{C}(8)-\mathrm{Fe}(1)-\mathrm{C}(6)$ & $68.6(3)$ \\
\hline $\mathrm{C}(9)-\mathrm{Fe}(1)-\mathrm{C}(6)$ & $69.5(3)$ \\
\hline $\mathrm{C}(1)-\mathrm{Fe}(1)-\mathrm{C}(6)$ & $155.7(3)$ \\
\hline $\mathrm{C}(3)-\mathrm{Fe}(1)-\mathrm{C}(6)$ & $125.5(3)$ \\
\hline $\mathrm{C}(7)-\mathrm{Fe}(1)-\mathrm{C}(10)$ & $68.3(3)$ \\
\hline $\mathrm{C}(5)-\mathrm{Fe}(1)-\mathrm{C}(10)$ & $156.2(3)$ \\
\hline $\mathrm{C}(2)-\mathrm{Fe}(1)-\mathrm{C}(10)$ & $124.6(3)$ \\
\hline $\mathrm{C}(4)-\mathrm{Fe}(1)-\mathrm{C}(10)$ & 121.2(3) \\
\hline $\mathrm{C}(8)-\mathrm{Fe}(1)-\mathrm{C}(10)$ & $68.7(3)$ \\
\hline $\mathrm{C}(9)-\mathrm{Fe}(1)-\mathrm{C}(10)$ & $41.1(3)$ \\
\hline $\mathrm{C}(1)-\mathrm{Fe}(1)-\mathrm{C}(10)$ & $161.4(3)$ \\
\hline $\mathrm{C}(3)-\mathrm{Fe}(1)-\mathrm{C}(10)$ & $106.8(3)$ \\
\hline $\mathrm{C}(6)-\mathrm{Fe}(1)-\mathrm{C}(10)$ & $41.2(3)$ \\
\hline $\mathrm{C}(33)-\mathrm{Fe}(2)-\mathrm{C}(35)$ & $119.4(3)$ \\
\hline $\mathrm{C}(33)-\mathrm{Fe}(2)-\mathrm{C}(32)$ & $40.1(3)$ \\
\hline $\mathrm{C}(35)-\mathrm{Fe}(2)-\mathrm{C}(32)$ & $109.5(3)$ \\
\hline $\mathrm{C}(33)-\mathrm{Fe}(2)-\mathrm{C}(36)$ & $104.9(3)$ \\
\hline $\mathrm{C}(35)-\mathrm{Fe}(2)-\mathrm{C}(36)$ & $41.9(3)$ \\
\hline $\mathrm{C}(32)-\mathrm{Fe}(2)-\mathrm{C}(36)$ & $125.7(3)$ \\
\hline $\mathrm{C}(33)-\mathrm{Fe}(2)-\mathrm{C}(31)$ & $68.3(3)$ \\
\hline $\mathrm{C}(35)-\mathrm{Fe}(2)-\mathrm{C}(31)$ & $128.2(3)$ \\
\hline $\mathrm{C}(32)-\mathrm{Fe}(2)-\mathrm{C}(31)$ & $40.4(3)$ \\
\hline $\mathrm{C}(36)-\mathrm{Fe}(2)-\mathrm{C}(31)$ & $164.5(3)$ \\
\hline
\end{tabular}




\begin{tabular}{|c|c|}
\hline $\mathrm{C}(33)-\mathrm{Fe}(2)-\mathrm{C}(30)$ & $68.1(3)$ \\
\hline $\mathrm{C}(35)-\mathrm{Fe}(2)-\mathrm{C}(30)$ & $165.5(3)$ \\
\hline $\mathrm{C}(32)-\mathrm{Fe}(2)-\mathrm{C}(30)$ & $67.4(3)$ \\
\hline $\mathrm{C}(36)-\mathrm{Fe}(2)-\mathrm{C}(30)$ & $151.6(3)$ \\
\hline $\mathrm{C}(31)-\mathrm{Fe}(2)-\mathrm{C}(30)$ & $40.3(3)$ \\
\hline $\mathrm{C}(33)-\mathrm{Fe}(2)-\mathrm{C}(29)$ & $40.5(3)$ \\
\hline $\mathrm{C}(35)-\mathrm{Fe}(2)-\mathrm{C}(29)$ & $153.0(3)$ \\
\hline $\mathrm{C}(32)-\mathrm{Fe}(2)-\mathrm{C}(29)$ & $67.4(3)$ \\
\hline $\mathrm{C}(36)-\mathrm{Fe}(2)-\mathrm{C}(29)$ & $116.3(3)$ \\
\hline $\mathrm{C}(31)-\mathrm{Fe}(2)-\mathrm{C}(29)$ & $68.1(3)$ \\
\hline $\mathrm{C}(30)-\mathrm{Fe}(2)-\mathrm{C}(29)$ & $40.5(3)$ \\
\hline $\mathrm{C}(33)-\mathrm{Fe}(2)-\mathrm{C}(38)$ & $161.3(3)$ \\
\hline $\mathrm{C}(35)-\mathrm{Fe}(2)-\mathrm{C}(38)$ & $68.2(3)$ \\
\hline $\mathrm{C}(32)-\mathrm{Fe}(2)-\mathrm{C}(38)$ & $157.5(3)$ \\
\hline $\mathrm{C}(36)-\mathrm{Fe}(2)-\mathrm{C}(38)$ & $68.6(3)$ \\
\hline $\mathrm{C}(31)-\mathrm{Fe}(2)-\mathrm{C}(38)$ & $122.2(3)$ \\
\hline $\mathrm{C}(30)-\mathrm{Fe}(2)-\mathrm{C}(38)$ & $108.9(3)$ \\
\hline $\mathrm{C}(29)-\mathrm{Fe}(2)-\mathrm{C}(38)$ & $125.2(3)$ \\
\hline $\mathrm{C}(33)-\mathrm{Fe}(2)-\mathrm{C}(37)$ & $122.5(3)$ \\
\hline $\mathrm{C}(35)-\mathrm{Fe}(2)-\mathrm{C}(37)$ & $69.3(3)$ \\
\hline $\mathrm{C}(32)-\mathrm{Fe}(2)-\mathrm{C}(37)$ & $160.7(3)$ \\
\hline $\mathrm{C}(36)-\mathrm{Fe}(2)-\mathrm{C}(37)$ & $40.5(2)$ \\
\hline $\mathrm{C}(31)-\mathrm{Fe}(2)-\mathrm{C}(37)$ & $154.9(3)$ \\
\hline $\mathrm{C}(30)-\mathrm{Fe}(2)-\mathrm{C}(37)$ & $118.6(3)$ \\
\hline $\mathrm{C}(29)-\mathrm{Fe}(2)-\mathrm{C}(37)$ & $104.5(3)$ \\
\hline $\mathrm{C}(38)-\mathrm{Fe}(2)-\mathrm{C}(37)$ & $41.4(2)$ \\
\hline $\mathrm{C}(33)-\mathrm{Fe}(2)-\mathrm{C}(34)$ & $155.1(3)$ \\
\hline $\mathrm{C}(35)-\mathrm{Fe}(2)-\mathrm{C}(34)$ & $39.9(3)$ \\
\hline $\mathrm{C}(32)-\mathrm{Fe}(2)-\mathrm{C}(34)$ & 123.1(3) \\
\hline $\mathrm{C}(36)-\mathrm{Fe}(2)-\mathrm{C}(34)$ & $68.8(3)$ \\
\hline $\mathrm{C}(31)-\mathrm{Fe}(2)-\mathrm{C}(34)$ & $111.2(3)$ \\
\hline $\mathrm{C}(30)-\mathrm{Fe}(2)-\mathrm{C}(34)$ & $128.8(3)$ \\
\hline $\mathrm{C}(29)-\mathrm{Fe}(2)-\mathrm{C}(34)$ & $164.2(3)$ \\
\hline $\mathrm{C}(38)-\mathrm{Fe}(2)-\mathrm{C}(34)$ & $40.7(3)$ \\
\hline $\mathrm{C}(37)-\mathrm{Fe}(2)-\mathrm{C}(34)$ & $69.1(2)$ \\
\hline$C(16)-P(1)-C(17)$ & $100.8(3)$ \\
\hline
\end{tabular}




$\begin{array}{lc}\mathrm{C}(16)-\mathrm{P}(1)-\mathrm{C}(23) & 108.0(3) \\ \mathrm{C}(17)-\mathrm{P}(1)-\mathrm{C}(23) & 107.0(3) \\ \mathrm{C}(16)-\mathrm{P}(1)-\mathrm{Pd}(1) & 118.5(3) \\ \mathrm{C}(17)-\mathrm{P}(1)-\mathrm{Pd}(1) & 115.5(2) \\ \mathrm{C}(23)-\mathrm{P}(1)-\mathrm{Pd}(1) & 106.4(2) \\ \mathrm{C}(44)-\mathrm{P}(2)-\mathrm{C}(45) & 107.8(3) \\ \mathrm{C}(44)-\mathrm{P}(2)-\mathrm{C}(51) & 103.0(3) \\ \mathrm{C}(45)-\mathrm{P}(2)-\mathrm{C}(51) & 107.6(3) \\ \mathrm{C}(44)-\mathrm{P}(2)-\mathrm{Pd}(2) & 119.0(2) \\ \mathrm{C}(45)-\mathrm{P}(2)-\mathrm{Pd}(2) & 104.4(2) \\ \mathrm{C}(51)-\mathrm{P}(2)-\mathrm{Pd}(2) & 114.4(2) \\ \mathrm{C}(9)-\mathrm{Pd}(1)-\mathrm{P}(1) & 88.8(2) \\ \mathrm{C}(9)-\mathrm{Pd}(1)-\mathrm{Cl}(1) & 94.6(2) \\ \mathrm{P}(1)-\mathrm{Pd}(1)-\mathrm{Cl}(1) & 171.86(7) \\ \mathrm{C}(9)-\mathrm{Pd}(1)-\mathrm{Cl}(2) & 172.8(2) \\ \mathrm{P}(1)-\mathrm{Pd}(1)-\mathrm{Cl}(2) & 96.79(6) \\ \mathrm{Cl}(1)-\mathrm{Pd}(1)-\mathrm{Cl}(2) & 80.39(5) \\ \mathrm{C}(37)-\mathrm{Pd}(2)-\mathrm{P}(2) & 87.80(18) \\ \mathrm{C}(37)-\mathrm{Pd}(2)-\mathrm{Cl}(1) & 173.27(18) \\ \mathrm{P}(2)-\mathrm{Pd}(2)-\mathrm{Cl}(1) & 95.74(6) \\ \mathrm{C}(37)-\mathrm{Pd}(2)-\mathrm{Cl}(2) & 96.44(18) \\ \mathrm{P}(2)-\mathrm{Pd}(2)-\mathrm{Cl}(2) & 172.38(7) \\ \mathrm{Cl}(1)-\mathrm{Pd}(2)-\mathrm{Cl}(2) & 80.70(6) \\ & \end{array}$

Symmetry transformations used to generate equivalent atoms: 
Table 4. Anisotropic displacement parameters $\left(\AA^{2} \times 10^{3}\right)$ for $\times r-452-1 \mathrm{c}$. The anisotropic displacement factor exponent takes the form: $-2 \pi^{2}\left[h^{2} a^{* 2} U^{11}+\ldots+2 h k a^{*} b^{*} U^{12}\right]$

\begin{tabular}{|c|c|c|c|c|c|c|}
\hline & $\mathrm{U}^{11}$ & $\mathrm{U}^{22}$ & $\mathrm{U}^{33}$ & $\mathrm{U}^{23}$ & $\mathrm{U}^{13}$ & $\mathrm{U}^{12}$ \\
\hline $\mathrm{C}(1)$ & $31(4)$ & $28(4)$ & $33(4)$ & $-9(3)$ & $0(4)$ & $-12(3)$ \\
\hline $\mathrm{C}(2)$ & $51(5)$ & $24(4)$ & $41(4)$ & $-15(4)$ & $32(4)$ & $-17(4)$ \\
\hline $\mathrm{C}(3)$ & $50(5)$ & $17(4)$ & $27(4)$ & $0(3)$ & $13(4)$ & $-2(3)$ \\
\hline $\mathrm{C}(4)$ & $45(5)$ & $19(4)$ & $39(4)$ & $-9(3)$ & $20(4)$ & $1(3)$ \\
\hline$C(5)$ & $42(5)$ & $31(4)$ & $31(4)$ & $-3(3)$ & $11(4)$ & $-7(4)$ \\
\hline $\mathrm{C}(6)$ & $19(4)$ & $30(4)$ & $34(4)$ & $3(3)$ & $10(3)$ & $-6(3)$ \\
\hline$C(7)$ & $42(5)$ & $18(4)$ & $36(4)$ & $5(3)$ & $16(4)$ & $-11(3)$ \\
\hline$C(8)$ & $36(4)$ & $11(3)$ & $23(4)$ & $-6(3)$ & $9(3)$ & $-4(3)$ \\
\hline $\mathrm{C}(9)$ & $37(4)$ & $22(3)$ & $18(3)$ & $-1(3)$ & $14(3)$ & $-10(3)$ \\
\hline$C(10)$ & $27(4)$ & $23(3)$ & $25(4)$ & $-4(3)$ & $10(3)$ & $-6(3)$ \\
\hline $\mathrm{C}(11)$ & $28(4)$ & $15(3)$ & $24(4)$ & $-8(3)$ & $6(3)$ & $-6(3)$ \\
\hline $\mathrm{C}(12)$ & $57(5)$ & $28(4)$ & $21(4)$ & $6(3)$ & $15(4)$ & $0(4)$ \\
\hline$C(13)$ & $48(5)$ & $27(4)$ & $27(4)$ & $9(4)$ & $0(4)$ & $9(4)$ \\
\hline $\mathrm{C}(14)$ & $32(4)$ & $24(4)$ & $31(4)$ & $11(3)$ & $3(4)$ & $9(3)$ \\
\hline$C(15)$ & $30(4)$ & $26(4)$ & $26(4)$ & $1(3)$ & $7(3)$ & $4(3)$ \\
\hline$C(16)$ & $39(4)$ & $15(3)$ & $18(4)$ & $1(3)$ & $-1(3)$ & $-2(3)$ \\
\hline $\mathrm{C}(17)$ & $24(4)$ & $24(4)$ & $20(4)$ & $-6(3)$ & $6(3)$ & $-3(3)$ \\
\hline $\mathrm{C}(18)$ & $28(4)$ & $24(4)$ & $24(4)$ & $0(3)$ & $7(3)$ & $-3(3)$ \\
\hline$C(19)$ & $27(4)$ & $27(4)$ & $40(4)$ & $-3(3)$ & $10(3)$ & $-4(3)$ \\
\hline$C(20)$ & $41(5)$ & $22(4)$ & $33(4)$ & $-13(3)$ & $-1(4)$ & $1(4)$ \\
\hline $\mathrm{C}(21)$ & $49(5)$ & $28(4)$ & $27(4)$ & $0(3)$ & $10(4)$ & $7(4)$ \\
\hline$C(22)$ & $46(5)$ & $23(4)$ & $23(4)$ & $-8(3)$ & $11(4)$ & $0(3)$ \\
\hline $\mathrm{C}(23)$ & $28(4)$ & $15(3)$ & $22(4)$ & $4(3)$ & $3(3)$ & $5(3)$ \\
\hline$C(24)$ & $27(4)$ & $23(4)$ & $47(5)$ & $9(4)$ & $14(4)$ & $4(3)$ \\
\hline$C(25)$ & $25(4)$ & $32(4)$ & $39(5)$ & $6(4)$ & $5(4)$ & $6(3)$ \\
\hline$C(26)$ & $27(4)$ & $44(5)$ & $38(4)$ & $6(4)$ & $10(3)$ & $-5(4)$ \\
\hline$C(27)$ & $41(5)$ & $38(5)$ & $48(5)$ & $-4(4)$ & $20(4)$ & $-6(4)$ \\
\hline$C(28)$ & $35(4)$ & $27(4)$ & $38(4)$ & $-10(4)$ & $4(4)$ & $-12(3)$ \\
\hline $\mathrm{C}(29)$ & $30(4)$ & $25(4)$ & $20(4)$ & $-10(3)$ & $1(3)$ & $-6(3)$ \\
\hline$C(30)$ & $25(4)$ & $24(4)$ & $28(4)$ & $2(3)$ & $-1(3)$ & $-10(3)$ \\
\hline$C(31)$ & $25(4)$ & $32(4)$ & $34(4)$ & $-4(4)$ & $14(3)$ & $-3(3)$ \\
\hline
\end{tabular}




\begin{tabular}{|c|c|c|c|c|c|c|}
\hline$C(32)$ & $43(5)$ & $32(4)$ & $24(4)$ & $-2(4)$ & $7(4)$ & $-17(4)$ \\
\hline$C(33)$ & $26(4)$ & $16(3)$ & $47(5)$ & $-5(3)$ & $3(4)$ & $-9(3)$ \\
\hline$C(34)$ & $25(4)$ & $20(3)$ & $20(3)$ & $-4(3)$ & $9(3)$ & $-3(3)$ \\
\hline$C(35)$ & $25(4)$ & $22(4)$ & $25(4)$ & $2(3)$ & $6(3)$ & $0(3)$ \\
\hline$C(36)$ & $19(3)$ & $20(3)$ & $18(3)$ & $-5(3)$ & $5(3)$ & $-4(3)$ \\
\hline$C(37)$ & $12(3)$ & $19(3)$ & $13(3)$ & $-6(3)$ & $1(3)$ & $-8(2)$ \\
\hline $\mathrm{C}(38)$ & $25(4)$ & $9(3)$ & $22(3)$ & $2(3)$ & $9(3)$ & $-2(3)$ \\
\hline$C(39)$ & $17(3)$ & $18(3)$ & $18(4)$ & $1(3)$ & $-1(3)$ & $-1(3)$ \\
\hline$C(40)$ & $23(4)$ & $24(4)$ & $19(4)$ & $-3(3)$ & $0(3)$ & $0(3)$ \\
\hline$C(41)$ & $26(4)$ & $25(4)$ & $24(4)$ & $13(3)$ & $1(3)$ & $-1(3)$ \\
\hline$C(42)$ & $24(4)$ & $22(4)$ & $33(4)$ & $1(3)$ & $1(3)$ & $3(3)$ \\
\hline$C(43)$ & $33(4)$ & $16(3)$ & $24(4)$ & $0(3)$ & $7(3)$ & $1(3)$ \\
\hline$C(44)$ & 21(4) & $18(3)$ & $12(4)$ & $3(3)$ & $-3(3)$ & $3(3)$ \\
\hline$C(45)$ & $28(4)$ & $22(4)$ & $16(3)$ & $-1(3)$ & $8(3)$ & $2(3)$ \\
\hline$C(46)$ & $33(4)$ & $25(4)$ & $23(4)$ & $-4(3)$ & $6(3)$ & $4(3)$ \\
\hline$C(47)$ & $35(4)$ & $22(4)$ & $24(4)$ & $-1(3)$ & $10(3)$ & $3(3)$ \\
\hline$C(48)$ & $50(5)$ & $20(4)$ & $29(4)$ & $-11(3)$ & $6(4)$ & $-7(3)$ \\
\hline$C(49)$ & $35(5)$ & $34(5)$ & $42(5)$ & $-18(4)$ & $6(4)$ & $-9(4)$ \\
\hline$C(50)$ & $26(4)$ & $28(4)$ & $38(5)$ & 1(4) & $11(4)$ & $3(3)$ \\
\hline$C(51)$ & $30(4)$ & $12(3)$ & $17(3)$ & $-1(3)$ & $5(3)$ & $1(3)$ \\
\hline$C(52)$ & $35(4)$ & $13(3)$ & $29(4)$ & $-4(3)$ & $8(3)$ & $-6(3)$ \\
\hline$C(53)$ & $39(4)$ & $31(4)$ & $29(4)$ & $-4(3)$ & $12(4)$ & $-5(4)$ \\
\hline$C(54)$ & $36(4)$ & $33(5)$ & $42(5)$ & $-2(4)$ & $13(4)$ & $-4(4)$ \\
\hline$C(55)$ & $43(5)$ & $39(5)$ & $37(5)$ & $-7(4)$ & $21(4)$ & $-13(4)$ \\
\hline$C(56)$ & $45(5)$ & $41(5)$ & $28(4)$ & $-7(4)$ & $19(4)$ & $-11(4)$ \\
\hline$C(57)$ & $36(5)$ & $26(4)$ & $42(5)$ & $4(4)$ & $4(4)$ & $2(4)$ \\
\hline$C(58)$ & $53(5)$ & $26(4)$ & $25(4)$ & $4(3)$ & $9(4)$ & $5(4)$ \\
\hline $\mathrm{Cl}(1)$ & $36(1)$ & $22(1)$ & $19(1)$ & $5(1)$ & $8(1)$ & $11(1)$ \\
\hline $\mathrm{Cl}(2)$ & $26(1)$ & 21(1) & 21(1) & $4(1)$ & $7(1)$ & $3(1)$ \\
\hline $\mathrm{Cl}(3)$ & $80(2)$ & $43(1)$ & $48(1)$ & $7(1)$ & $29(1)$ & $9(1)$ \\
\hline $\mathrm{Cl}(4)$ & $50(1)$ & $46(1)$ & $49(1)$ & $2(1)$ & $22(1)$ & $8(1)$ \\
\hline $\mathrm{Cl}(5)$ & $42(1)$ & $46(1)$ & $105(2)$ & $-14(2)$ & $5(1)$ & $0(1)$ \\
\hline $\mathrm{Cl}(6)$ & $39(1)$ & $36(1)$ & $79(2)$ & $-11(1)$ & $7(1)$ & $3(1)$ \\
\hline $\mathrm{Cl}(7)$ & $41(1)$ & $38(1)$ & $38(1)$ & $-7(1)$ & 11(1) & $3(1)$ \\
\hline $\mathrm{Cl}(8)$ & 192(4) & $48(2)$ & $43(2)$ & $2(1)$ & $56(2)$ & $14(2)$ \\
\hline $\mathrm{Fe}(1)$ & $28(1)$ & $20(1)$ & $21(1)$ & $-1(1)$ & $9(1)$ & $-2(1)$ \\
\hline
\end{tabular}




\begin{tabular}{lllllll}
$\mathrm{Fe}(2)$ & $23(1)$ & $16(1)$ & $19(1)$ & $-2(1)$ & $7(1)$ & $-2(1)$ \\
$\mathrm{P}(1)$ & $22(1)$ & $15(1)$ & $19(1)$ & $1(1)$ & $3(1)$ & $2(1)$ \\
$\mathrm{P}(2)$ & $24(1)$ & $14(1)$ & $17(1)$ & $1(1)$ & $4(1)$ & $1(1)$ \\
$\operatorname{Pd}(1)$ & $24(1)$ & $14(1)$ & $17(1)$ & $2(1)$ & $4(1)$ & $2(1)$ \\
$\operatorname{Pd}(2)$ & $23(1)$ & $13(1)$ & $16(1)$ & $1(1)$ & $5(1)$ & $2(1)$ \\
\hline
\end{tabular}


Table 5. Hydrogen coordinates $\left(\mathrm{x} 10^{4}\right)$ and isotropic displacement parameters $\left(\AA^{2} \times 10^{3}\right)$ for $\mathrm{xr}-452-1 \mathrm{c}$.

\begin{tabular}{|c|c|c|c|c|}
\hline & $\mathrm{x}$ & $\mathrm{y}$ & $\mathrm{z}$ & $\mathrm{U}(\mathrm{eq})$ \\
\hline $\mathrm{H}(1)$ & 4699 & 8170 & 6782 & 40 \\
\hline $\mathrm{H}(2)$ & 4067 & 8979 & 5232 & 41 \\
\hline $\mathrm{H}(3)$ & 2340 & 9728 & 5110 & 38 \\
\hline $\mathrm{H}(4)$ & 1934 & 9355 & 6606 & 39 \\
\hline $\mathrm{H}(5)$ & 3359 & 8426 & 7600 & 42 \\
\hline $\mathrm{H}(6)$ & 720 & 7488 & 5699 & 33 \\
\hline $\mathrm{H}(7)$ & 2257 & 6580 & 6579 & 37 \\
\hline $\mathrm{H}(10)$ & 972 & 7969 & 4151 & 30 \\
\hline $\mathrm{H}(12)$ & 3694 & 5887 & 6985 & 42 \\
\hline $\mathrm{H}(13)$ & 5227 & 5038 & 7517 & 45 \\
\hline $\mathrm{H}(14)$ & 6267 & 4878 & 6533 & 37 \\
\hline $\mathrm{H}(15)$ & 5879 & 5645 & 5152 & 34 \\
\hline $\mathrm{H}(17)$ & 4557 & 5219 & 3554 & 27 \\
\hline $\mathrm{H}(18 \mathrm{~A})$ & 2388 & 5362 & 3350 & 31 \\
\hline $\mathrm{H}(18 \mathrm{~B})$ & 3246 & 4961 & 4256 & 31 \\
\hline $\mathrm{H}(19 \mathrm{~A})$ & 3602 & 3810 & 3334 & 38 \\
\hline H(19B) & 2388 & 3802 & 3240 & 38 \\
\hline $\mathrm{H}(20 \mathrm{~A})$ & 1970 & 4513 & 1798 & 43 \\
\hline $\mathrm{H}(20 \mathrm{~B})$ & 2626 & 3644 & 1762 & 43 \\
\hline $\mathrm{H}(21 \mathrm{~A})$ & 4152 & 4481 & 1951 & 43 \\
\hline $\mathrm{H}(21 \mathrm{~B})$ & 3241 & 4897 & 1093 & 43 \\
\hline $\mathrm{H}(22 \mathrm{~A})$ & 4188 & 6022 & 2150 & 37 \\
\hline $\mathrm{H}(22 \mathrm{~B})$ & 2974 & 6035 & 2056 & 37 \\
\hline $\mathrm{H}(23)$ & 5093 & 7453 & 3216 & 27 \\
\hline $\mathrm{H}(24 \mathrm{~A})$ & 6502 & 6122 & 4214 & 38 \\
\hline $\mathrm{H}(24 \mathrm{~B})$ & 5810 & 6048 & 3129 & 38 \\
\hline $\mathrm{H}(25 \mathrm{~A})$ & 7498 & 6595 & 3245 & 40 \\
\hline $\mathrm{H}(25 \mathrm{~B})$ & 6635 & 7325 & 2773 & 40 \\
\hline $\mathrm{H}(26 \mathrm{~A})$ & 8036 & 7977 & 3930 & 44 \\
\hline $\mathrm{H}(26 \mathrm{~B})$ & 7885 & 7307 & 4701 & 44 \\
\hline
\end{tabular}




\begin{tabular}{|c|c|c|c|c|}
\hline $\mathrm{H}(27 \mathrm{~A})$ & 7080 & 8662 & 4854 & 49 \\
\hline $\mathrm{H}(27 \mathrm{~B})$ & 6403 & 8646 & 3765 & 49 \\
\hline $\mathrm{H}(28 \mathrm{~A})$ & 6165 & 7466 & 5167 & 42 \\
\hline $\mathrm{H}(28 \mathrm{~B})$ & 5331 & 8190 & 4622 & 42 \\
\hline $\mathrm{H}(29)$ & 3353 & 10280 & 1063 & 32 \\
\hline $\mathrm{H}(30)$ & 4523 & 9030 & 910 & 33 \\
\hline $\mathrm{H}(31)$ & 4494 & 8964 & -778 & 35 \\
\hline $\mathrm{H}(32)$ & 3336 & 10193 & -1644 & 41 \\
\hline $\mathrm{H}(33)$ & 2593 & 10973 & -538 & 38 \\
\hline $\mathrm{H}(34)$ & 2280 & 7680 & -1462 & 25 \\
\hline $\mathrm{H}(35)$ & 1161 & 8989 & -2118 & 29 \\
\hline $\mathrm{H}(38)$ & 2533 & 7591 & 284 & 22 \\
\hline $\mathrm{H}(40)$ & 559 & 10345 & -2137 & 28 \\
\hline $\mathrm{H}(41)$ & -519 & 11547 & -2416 & 32 \\
\hline $\mathrm{H}(42)$ & -1354 & 11966 & -1361 & 34 \\
\hline $\mathrm{H}(43)$ & -1054 & 11173 & 45 & 30 \\
\hline $\mathrm{H}(45)$ & 709 & 10329 & 2472 & 26 \\
\hline $\mathrm{H}(46 \mathrm{~A})$ & -461 & 11707 & 1337 & 33 \\
\hline $\mathrm{H}(46 \mathrm{~B})$ & -970 & 10976 & 1827 & 33 \\
\hline $\mathrm{H}(47 \mathrm{~A})$ & 117 & 11426 & 3318 & 33 \\
\hline $\mathrm{H}(47 \mathrm{~B})$ & -560 & 12244 & 2776 & 33 \\
\hline $\mathrm{H}(48 \mathrm{~A})$ & 874 & 12775 & 2348 & 42 \\
\hline $\mathrm{H}(48 \mathrm{~B})$ & 1233 & 12660 & 3465 & 42 \\
\hline $\mathrm{H}(49 \mathrm{~A})$ & 2088 & 11331 & 3373 & 46 \\
\hline $\mathrm{H}(49 \mathrm{~B})$ & 2508 & 12071 & 2822 & 46 \\
\hline $\mathrm{H}(50 \mathrm{~A})$ & 1419 & 11531 & 1356 & 36 \\
\hline $\mathrm{H}(50 \mathrm{~B})$ & 2100 & 10725 & 1921 & 36 \\
\hline $\mathrm{H}(51)$ & -1548 & 9666 & 606 & 24 \\
\hline $\mathrm{H}(52 \mathrm{~A})$ & -1107 & 8569 & -344 & 31 \\
\hline $\mathrm{H}(52 \mathrm{~B})$ & -564 & 7983 & 567 & 31 \\
\hline $\mathrm{H}(53 \mathrm{~A})$ & -2761 & 8378 & -145 & 39 \\
\hline $\mathrm{H}(53 \mathrm{~B})$ & -2262 & 7426 & -174 & 39 \\
\hline $\mathrm{H}(54 \mathrm{~A})$ & -1760 & 7316 & 1458 & 44 \\
\hline $\mathrm{H}(54 \mathrm{~B})$ & -2984 & 7524 & 1070 & 44 \\
\hline $\mathrm{H}(55 \mathrm{~A})$ & -2669 & 9028 & 1490 & 45 \\
\hline $\mathrm{H}(55 \mathrm{~B})$ & -2145 & 8434 & 2399 & 45 \\
\hline
\end{tabular}




\begin{tabular}{lrrrr}
$\mathrm{H}(56 \mathrm{~A})$ & -958 & 9547 & 2262 & 43 \\
$\mathrm{H}(56 \mathrm{~B})$ & -488 & 8591 & 2189 & 43 \\
$\mathrm{H}(57)$ & 5080 & 5910 & 9789 & 44 \\
$\mathrm{H}(58)$ & 1197 & 5584 & 4840 & 43 \\
\hline
\end{tabular}




\section{References}

1. Little, W. F.; Reilley, C. N.; Johnson, J. D.; Lynn, K. N. J. Am. Chem. Soc. 1964, 86, 1376.

2. Stevens, A. M.; Richards, C. J. Organometallics, 1999, 18, 1346.

3. Prasad, R. S.; Anderson, C. E.; Richards, C. J.; Overman, L. E. Organometallics, in press.

4. Collect Data collection and processing user interface: Collect: Data collection software, R. Hooft, Nonius B.V., 1998).

5. Denzo Data collection and processing software: Z. Otwinowski and W. Minor, Methods in Enzymology, Volume 276: Macromolecular Crystallography, part A, p.307-326, 1997, C.W. Carter, Jr and R.M. Sweet, Eds., Academic Press.

6. maXus solution and refinement software suite: S. Mackay, C.J. Gilmore, C. Edwards, M. Tremayne, N. Stewart, K. Shankland. maXus: a computer program for the solution and refinement of crystal structures from diffraction data.

7. Sheldrick, G. M. (2003). SADABS - Bruker Nonius area detector scaling and absorption correction - V2.10.

8. DIRDIF99 program system. P. T. Beurskens, G. Beurskens, W. P. Bosman, R. de Gelder, S. Garcia-Granda, R. O. Gould, R. Israel and J. M. M. Smits, Crystallography Laboratory, University of Nijmegen, The Netherlands. 1999.

9. SHELX97 [Includes SHELXS97, SHELXL97, CIFTAB (and SHELXA) ] - Programs for Crystal Structure Analysis (Release 97-2). G. M. Sheldrick, Institüt für Anorganische Chemie der Universität, Tammanstrasse 4, D-3400 Göttingen, Germany, 1998.

10. ORTEP3 for Windows - Farrugia, L. J. J. Appl. Crystallogr. 1997, 30, 565.

11. Spek, A. L. (1998) PLATON, A Multipurpose Crystallographic Tool, Utrecht University, Utrecht, The Netherlands.

12. WINGX, Farrugia, L. J. J. Appl. Cryst. 1999, 32, 837. 